\title{
A New Method for Treating Wells in Reservoir Simulation
}

Gregory M. Gessel

Brigham Young University - Provo

Follow this and additional works at: https://scholarsarchive.byu.edu/etd

Part of the Chemical Engineering Commons

\section{BYU ScholarsArchive Citation}

Gessel, Gregory M., "A New Method for Treating Wells in Reservoir Simulation" (2007). Theses and Dissertations. 1405.

https://scholarsarchive.byu.edu/etd/1405

This Thesis is brought to you for free and open access by BYU ScholarsArchive. It has been accepted for inclusion in Theses and Dissertations by an authorized administrator of BYU ScholarsArchive. For more information, please contact scholarsarchive@byu.edu, ellen_amatangelo@byu.edu. 


\section{A NEW METHOD FOR TREATING WELLS \\ IN RESERVOIR SIMULATION}

by

Gregory M. Gessel

A thesis submitted to the faculty of Brigham Young University

In partial fulfillment of the requirements for the degree of

Master of Science

Department of Chemical Engineering

Brigham Young University

August 2007 



\title{
BRIGHAM YOUNG UNIVERSITY
}

\section{GRADUATE COMMITTEE APPROVAL}

\author{
of a thesis submitted by \\ Gregory M. Gessel
}

This dissertation has been read by each member of the following graduate committee and by majority vote has been found to be satisfactory.

Date

Date

Date
Hugh B. Hales, Chair

Larry L. Baxter

Ronald E. Terry 



\section{BRIGHAM YOUNG UNIVERSITY}

As chair of the candidate's graduate committee, I have read the thesis of Gregory M. Gessel in its final form and have found that (1) its format, citations, and bibliographical style are consistent and acceptable and fulfill university and department style requirements: (2) its illustrative materials including figures, tables, and charts are in place; and (3) the final manuscript is satisfactory to the graduate committee and is ready for submission to the university library.

Date

Accepted for the Department
Hugh B. Hales

Chair, Graduate Committee
Larry L. Baxter

Graduate Coordinator

Accepted for the College

\begin{tabular}{l}
\hline Alan R. Parkinson \\
Dean, Ira A. Fulton College of Engineering \\
and Technology
\end{tabular}





\begin{abstract}
A NEW METHOD FOR TREATING WELLS

IN RESERVOIR SIMULATION
\end{abstract}

\author{
Gregory M. Gessel \\ Department of Chemical Engineering \\ Master of Science
}

A new method for formulating finite difference equations for reservoir simulation has been developed. It can be applied throughout the entire simulated reservoir or to local segments. When applied to cells containing vertical, fully penetrating, straight-line wells in a homogeneous reservoir, the resulting equations are equivalent to Peaceman's classical well equations used in most reservoir simulators today. However, when the new finite difference equations are applied to both the well-containing cells, and their neighbors, the accuracy of the simulation improves substantially. The method produces still better accuracy results when applied throughout the reservoir. Unlike the Peaceman correction, the new method also applies to reservoirs containing wells of complex geometry. This includes wells that are closely spaced and wells near reservoir faults and external boundaries. 

The method results from the incorporation of approximate analytical expressions for the pressure into the reservoir simulator's finite difference equations. By incorporating the "physics" of the flow into the solution, rather than relying on polynomial-based finite difference equations based on Taylor's series, as is usually done, solution accuracy improves. Accuracy is particularly improved around the wells where near-singularities in the pressure occur. Polynomials are incapable of accurately representing singularities. 



\section{ACKNOWLEDGMENTS}

I would like express appreciation for Dr. Hugh B. Hales for all of the help and guidance he provided as my advisor. I would also like to thank the other members of my

graduate committee, Dr. Larry L. Baxter and Dr. Ronald E. Terry for their input and willingness to serve on my committee. I am also very grateful for the financial support that the International Reservoir Simulation Research Institute has provided. 



\section{TABLE OF CONTENTS}

$\underline{\text { Section }} \quad \underline{\text { Page }}$

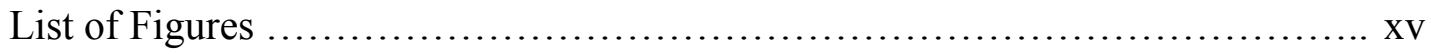

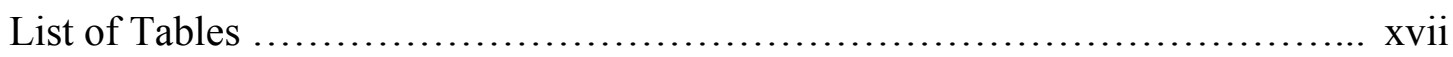

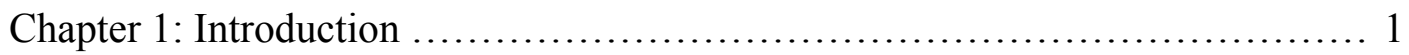

Chapter 2: Background and Derivation of New Method ..................... 7

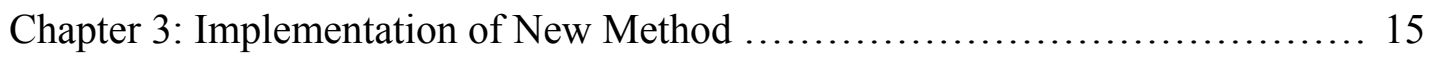

Chapter 4: Results ................................................ 17

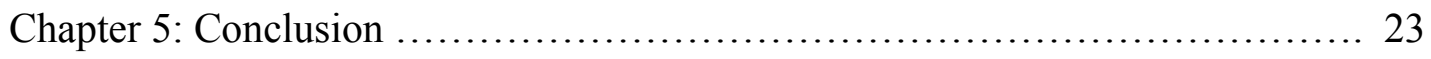

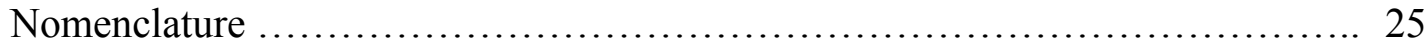

References ....................................................... 27

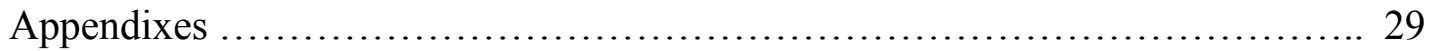

Appendix A ................................................... 31

Appendix B ................................................... 55 


\section{LIST OF FIGURES}

$\underline{\text { Figure }}$

$\underline{\text { Page }}$

1. Weber et al. Results ................................................ 4

2. Comparison of Weber's and Composite Solution Results ................... 17

3. One Well and Two Well results at varying patch sizes ..................... 19

4. Effect of well location in full grid composite solution ..................... 20

5. Results of well placement for one-cell composite solution $\ldots \ldots \ldots \ldots \ldots \ldots \ldots \ldots 21$

6. Errors of Peaceman and Composite solutions for offcenter wells .............. 22

A-1. Model Reservoir with point source wells ........................... 37

A-2. Example of area used to calculate the linking permeability between

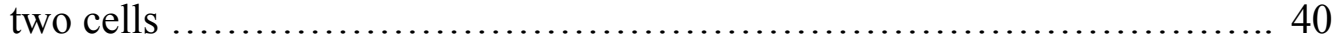

A-3. Upscaling results ............................................ 41

A-4. Horizontal layer of reservoir with fully-penetrating, straight-line wells ...... 45

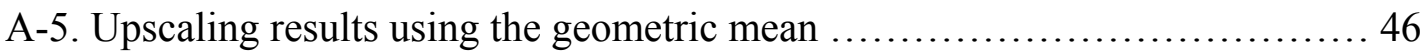

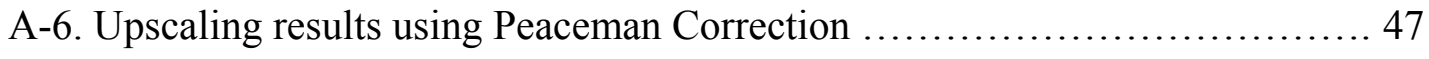

A-7. Upscaling results using the Integral Method $\ldots \ldots \ldots \ldots \ldots \ldots \ldots \ldots \ldots \ldots \ldots$

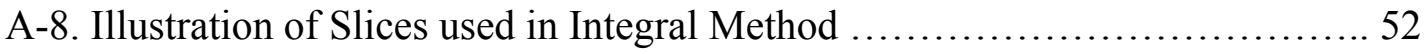

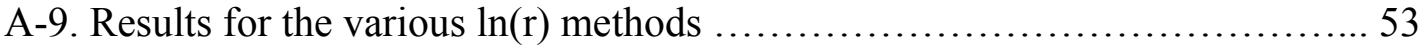




\section{LIST OF TABLES}

$\underline{\text { Table }}$

$\underline{\text { Page }}$

A-1. Constants used in equations (14)-(20)

54

xvii 
xviii 


\section{Chapter 1}

\section{Introduction}

Reservoir simulators are important tools in the petroleum industry. Simulators predict production rates and volumes of oil, gas, and water. Sometimes they also predict hydrocarbon compositions in each phase. This involves solving for the pressure and the saturations (i.e. fluid volume fractions) of the oil, gas and water phases in the reservoir. Traditional finite difference methods describe the pressure everywhere except around the wells. The grid-block size is generally many times larger than the well bore. This causes the wells to create near-singularities in pressure which are not handled well by finite difference equations because of their basis on polynomials. Many simulators use an empirical productivity index $(P I)$ to correct simulated well cell pressures. The

productivity index relates the flow rate $(Q)$ to the pressures of the well and the cell $(P)$.

$$
Q=P I *\left(P_{\text {well }}-P_{\text {cell }}\right)
$$

In 1978, Peaceman ${ }^{1}$ presented a method of calculating the productivity index. He found that the well cell's pressure can be approximated as the actual flowing pressure at a radial distance of $0.2 \Delta x$ from the well. 


$$
P_{\text {well }}-P_{\text {cell }}=\frac{1}{2 \pi} \frac{Q \mu}{K h} \ln \left(\frac{0.2 \Delta x}{r}\right)
$$

where $K$ is the permeability and $h$ is the reservoir thickness.

This result is based on the pressures in a 2-D, homogenous, isotropic reservoir with the wells in a square five-spot pattern, each centered in a square grid block. This Peaceman correction is still widely used to calculate well bore pressures. However, errors occur when the well geometry and reservoir properties differ from those of the original investigation.

Peaceman $^{2}$ subsequently demonstrated the applicability of his earlier well model ${ }^{1}$ for multiple wells in the same cells and for single wells that are not centered in cells as long as the wells are far from all other wells and far from the reservoir boundaries. It seems intuitively satisfying that well pressures do not change much as they move small intra-cell distances if the wells are isolated from the boundaries and other wells. However, one would expect the pressures in neighboring cells, and the fluxes through the well cell's boundaries to vary substantially. Had Peaceman reported these values, errors would likely have been substantial. Similarly wells within the same cell are likely to maintain their relative pressures if their spacing is maintained. However, well cell fluxes and neighboring cell pressures will depend on the location of these wells within the cell.

Kunianksy and Hillestad ${ }^{3}$ also found that Peaceman's equivalent radius of 0.2 times the length of the grid block is a good approximation for wells that are not centered in the cell. They investigated wells located on the edge or the corner of a block. 
Abou-Kassam and $\mathrm{Aziz}^{6}$ developed an analytical method for computing the PI in grid blocks that are square or rectangular. The method is restricted to 2-D, single phase models. In deriving the method there is also the assumption of radial flow, which is not always valid.

Williamson and Chappelear ${ }^{4,5}$ addressed the problem of coupling that occurs between the wellbore and the reservoir. Because the wellbore is so small compared to the grid block size it can not be described as a boundary. They instead use a well model that approximates the well boundary by a source function. Source functions were derived for many conditions including incompressible single phase flow, compressible single phase flow and multiphase flow.

Ding et al. ${ }^{7}$ took a somewhat different approach. They proposed altered transmissiblities between the well cell and neighboring cells, as a companion to the well equation. This was done using a control volume method that allowed for the modeling of distorted grid blocks. The method is used in a limited area surrounding each well and can be used in anisotropic and heterogeneous media.

Weber et al. ${ }^{8}$ took a substantially different approach. They abandoned the time honored finite difference equations resulting from the 1715 work of Brook Taylor ${ }^{9}$, father of finite difference mathematics. Instead of using Taylor's series as the basis for their finite difference equations, they built finite-difference equations based on the physics of the flow around the wells. Weber et al. incorporated $\ln (\mathrm{r})$-terms in their finite difference terms for the pressure derivatives consistent with the theoretical pressure profiles around straight line wells in an infinite system. They also used 1/r-terms consistent with the theoretical pressure profiles around point sources. 
Figure 1 shows the Weber et al.'s pressure errors resulting from flow between two six-inch diameter, straight line, vertical wells (bhp's $\pm 1,000 \mathrm{psi}$ ) located in the center of square reservoir segments, such that the over-all dimensions of the reservoir was $900 \mathrm{ft}$ by $1800 \mathrm{ft}$.

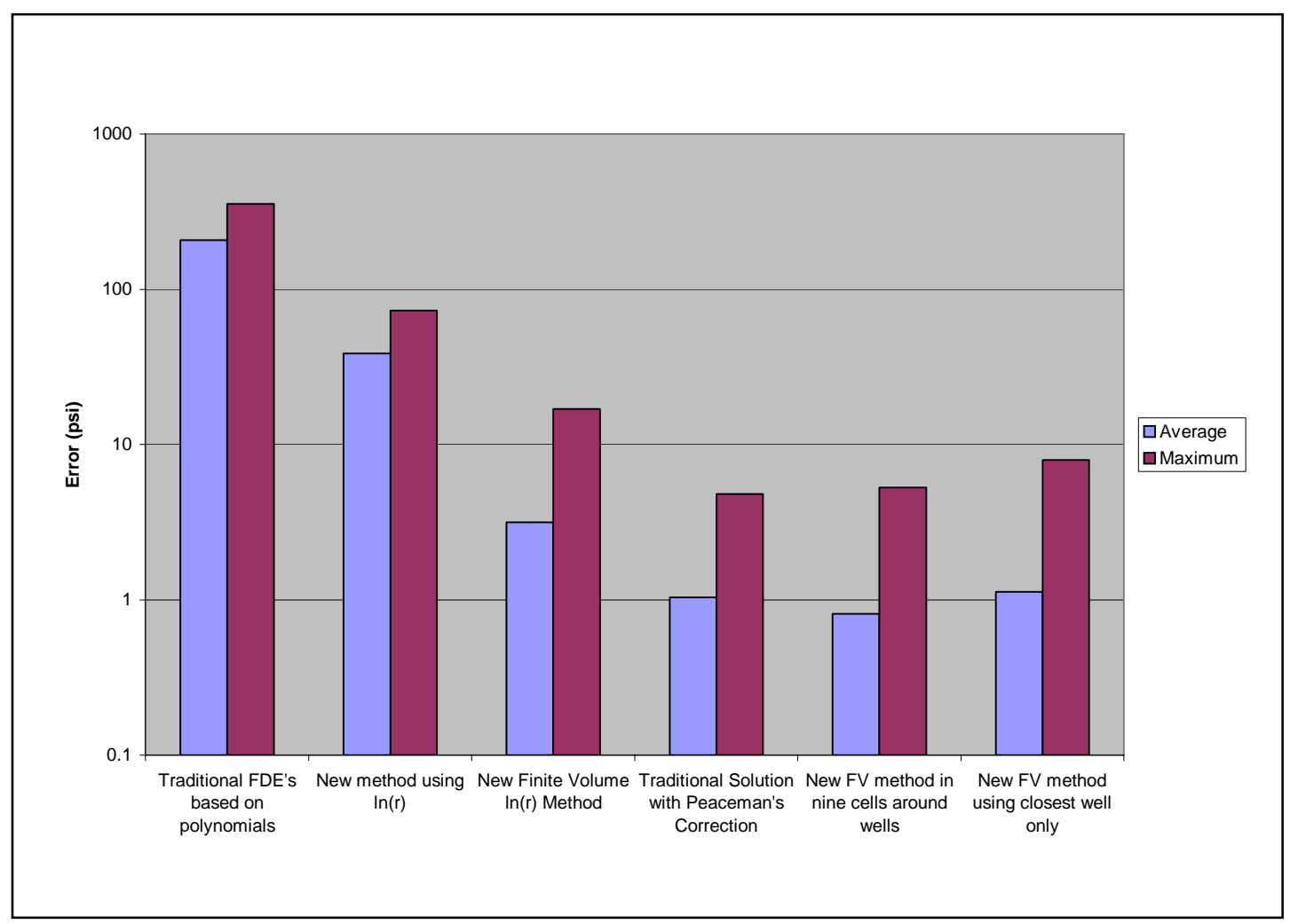

Figure 1. Weber et al. ${ }^{8}$ Results

It shows that the pressure errors were improved about a hundred fold relative to an uncorrected well treatment. However, the new method was no more accurate than Peaceman's correction. In a similar geometry, but using point source wells, Weber et al. found a thousand fold improvement in accuracy relative to the uncorrected case, and a hundred fold improvement relative to Peaceman's correction. These results suggest that 
Weber's new formulation gives about the same accuracy as Peaceman, in geometries in which Peaceman applies. However, this high accuracy is maintained, or even improved, in other geometries in which Peaceman's correlation works poorly. Inclusion of the physics in the finite difference equations can considerably increase the accuracy of the solution.

The traditional finite difference formulation of the simplified pressure equation used by Weber et al. is second order accurate. When $\ln (\mathrm{r})$ and $1 / \mathrm{r}-$ terms replace the quadratic terms the resulting equations do a good job around the wells. However, they are not as accurate near the no-flow reservoir boundaries. This is because the traditional equation can approximate the boundary condition $\mathrm{dp} / \mathrm{dx}=0$ whereas $\log (\mathrm{r})$ and $1 / \mathrm{r}$ cannot.

This thesis describes the use of finite difference equations which include $\ln (r)$ terms and quadratic terms without increasing the number of pressure points in the finite difference equations nor the complexity of the resulting linear algebra. The pressure equation remains second order accurate while accurately representing the near singularities around the wells. 


\section{Chapter 2}

\section{Background and Derivation of Composite Method}

The new, composite method better handles wells by not relying on Taylor-seriesbased finite difference equations. New approximate analytical expressions for the pressure are incorporated into the simulators finite difference equations, which results in much better solutions. These finite difference equations produce numerical solutions to the partial differential equations.

Laplace's equation represents the pressure in a homogeneous, isotropic reservoir at steady state:

$$
\Delta^{2} P=0
$$

In two-dimensional, Cartesian coordinates, Laplace's equation is

$$
\frac{\partial^{2} P}{\partial x^{2}}+\frac{\partial^{2} P}{\partial y^{2}}=0
$$

The traditional method of developing finite difference equations approximates the partial derivative using a Taylor series expansion of a function about a point. 


$$
P(x)=P(a)+\sum_{n=0} \frac{P^{n}(a)}{n !}(x-a)^{n}
$$

where $P^{n}(a)$ is the n-th derivative of $P$ at $a$.

There are many ways to construct finite-difference approximations to the derivatives in this fashion, but Laplace's equation is particularly suited to a centered difference form that uses three points in each direction and uniform grid spacing. This is because the resulting finite difference equations are second-order accurate in $\Delta \mathrm{x}$. Such Taylor-series-derived finite-difference approximations of the Laplace equation are:

$$
\frac{\partial^{2} P}{\partial x^{2}}=\frac{P_{j+1}-2 P_{j}+P_{j-1}}{\Delta x^{2}}
$$

Exactly the same results are obtained if $\mathrm{P}$ is assumed to be a quadratic:

$$
P(x)=a x^{2}+b x+c
$$

If the following three points are substituted into equation (7):

$$
\begin{aligned}
& P(\Delta x)=p_{i+1} \\
& P(0)=p_{i} \\
& P(-\Delta x)=p_{i-1}
\end{aligned}
$$


and the resulting three equations are solved for the parameters $a, \mathrm{~b}$, and $\mathrm{c}$, (only a is needed in this case) an expression that is identical to the Taylor Series derived form results:

$$
\frac{\partial^{2} P}{\partial x^{2}}=2 a=\frac{p_{i+1}-2 p_{i}+p_{i-1}}{\Delta x^{2}}
$$

Alternatively one can assume that the solution is piecewise linear, that is, between any two points it is expressed as

$$
P(x)=b x+c
$$

Then

$$
\left.\frac{d P}{d x}\right|_{i+1 / 2}=\frac{P_{i+1}-P_{i}}{\Delta x} \text { and }\left.\frac{d P}{d x}\right|_{i-1 / 2}=\frac{P i-P_{i-1}}{\Delta x}
$$

And

$$
\frac{\partial^{2} P}{\partial x^{2}}=\frac{1}{\Delta x}\left(\left.\frac{d P}{d x}\right|_{i+\frac{1}{2}}-\left.\frac{d P}{d x}\right|_{i-\frac{1}{2}}\right)=\frac{P_{j+1}-2 P_{j}+P_{j-1}}{\Delta x^{2}}
$$


Similar derivations can be made based on expressions with greater physical relevance. For example, the pressure around a straight line well in an infinite, homogeneous reservoir is given by ${ }^{23}$

$$
P=P_{w}+\frac{Q \mu}{2 \pi K} \ln \left(\frac{r}{r_{w}}\right)
$$

where $r$ is the perpendicular distance from the well. In analogy with equation (10), Weber et al. ${ }^{8}$ assumed:

$$
P=a \sum_{n}^{\substack{\text { all } \\ \text { wells }}} Q \ln \left(r_{n}\right)+c
$$

Resulting in

$$
\left.\frac{\partial P}{\partial x}\right|_{i+\frac{1}{2}}=\frac{\left(P_{i+1}-P_{i}\right) \sum \frac{Q x_{i+1 / 2}}{r_{i+1 / 2}^{2}}}{\sum Q \ln \left(r_{i+1}\right)-\sum Q \ln \left(r_{i}\right)}
$$


Weber's results appear in Figure 1, and were discussed previously. Although his method shows promise of wider applicability than Peaceman's ${ }^{1}$ classical method, it showed no greater accuracy for the simple geometry that Weber investigated.

Several other basis functions which included $\ln (r)$ terms were investigated as part of this work. Finite difference equations that use only three points in each direction lend themselves to rapid matrix inversion schemes and provide spatial flexibility in describing wells . It appears that although the $\ln (r)$ FDE's accurately represent the pressure around the wells, they do not do as good a job in the more distant areas as the traditional secondorder accurate FDE's based on Taylor's series.

In accordance with this rationale, the following basis function was considered:

$$
P=\sum_{n}^{\substack{\text { all } \\ \text { wells }}} \frac{Q_{n} \mu}{2 \pi K} \ln \left(r_{n}\right)+a x^{2}+b x+c
$$

It provides the logarithmic function's accuracy around the wells, and the second-order accuracy of the polynomial-based FDE's in the rest of the reservoir. The resulting solution includes two parts:

$$
P=P_{a}+P_{f}
$$


where $P_{a}$ is the analytical solution and $P_{f}$ is the finite difference solution. The finite difference component represents the error in the analytical solution. $P_{a}$ satisfies the Laplace equation exactly if coefficients are constant, there are no boundaries, and the reservoir has other ideal features. Hence the finite difference component is of exactly the same traditional form. However, $P_{a}$ includes all of the information on the well fluxes. $P_{f}$ has no wells in this sense. Instead it has fluxes through the reservoir boundaries. $P_{a}$ describes an infinite system and therefore results in fluxes through the reservoir boundaries that do not exist in the actual system. $P_{f}$ compensates for them. The $P_{f}$ boundary condition becomes

$$
\begin{gathered}
\frac{\mathrm{d}}{\mathrm{a}}=0 \\
\Rightarrow \frac{\mathrm{P}_{\mathrm{f}}}{\mathrm{a}}=\frac{\mathrm{dP}_{\mathrm{a}}}{\mathrm{d}}
\end{gathered}
$$

where $n$ is the direction normal to the boundary. (When $P_{a}$ is substituted into the more general pressure equation, small residuals result that must be eliminated by the finite difference component.)

Weber et al. demonstrated that the finite difference equations did not have to be the same throughout the reservoir. He used the $\ln (r)$-based equation around the well and traditional Taylor series based equations elsewhere in a hybrid fashion. He found that $\ln (\mathrm{r})$ equations used only in the well cells and their immediate neighbors provided the greatest accuracy. In this work, the composite analytical and finite difference solution throughout the reservoir gave the best results. Nevertheless, this composite form can also be applied 
in a hybrid fashion. When the composite FDE's were used only in the well cells, results identical to Peaceman's solution resulted. This suggests that the composite solution can provide Peaceman-like results for all well geometries, including wells not centered in the finite difference cells, wells that pass through the cells at an angle, and wells of limited completion interval. Details of these results follow. 


\section{Chapter 3}

\section{Implementation of New Method}

The new method was implemented using Microsoft Excel. The two parts of the composite solution are calculated in separate groups of cells and then combined. This total pressure was then compared to Morel-Seytoux's ${ }^{10}$ analytical solution, and the maximum, minimum and absolute errors are calculated.

If a contiguous group of cells, or a "patch" of cells smaller than the entire reservoir is selected, it is not necessary to calculate the analytical solution everywhere. It is calculated in the cells of the patch and their neighbors. So for the one-cell patch the analytical solution is calculated in the well cell and the four adjoining cells.

The cells in the finite difference component of the solution contain traditional finite difference equations if they are outside the patch and its neighbors. Cells in the patch have finite difference equations that also include the analytical solution. The cells surrounding the patch have finite difference equations that are modified with the new boundary condition that ensures there is no flow at the reservoir boundaries.

The total pressure is calculated by combining the two solutions. The pressure of cells that lie outside the patch is given by the results of the FDE component. For cells that lie in the patch the pressure is calculated by adding the FDE component and the analytical component. Morel-Seytoux's analytical solution is subtracted from this total solution and the errors are calculated. The minimum and maximum errors are simply 
found in the cells with the smallest and largest deviation. The absolute error is the average of the absolute value of the error in each cell.

In the one-cell and full reservoir patch files the horizontal position of the wells can be specified which allows for wells that are not centered in the cells. MorelSeytoux.xls provides the analytical solution for the given well separation. 


\section{Chapter 4}

\section{Results}

Figure 2 shows the results of Weber et al. ${ }^{8}$ in the first four column pairs. The results from the new composite solution method are appended as the right-most column pair.

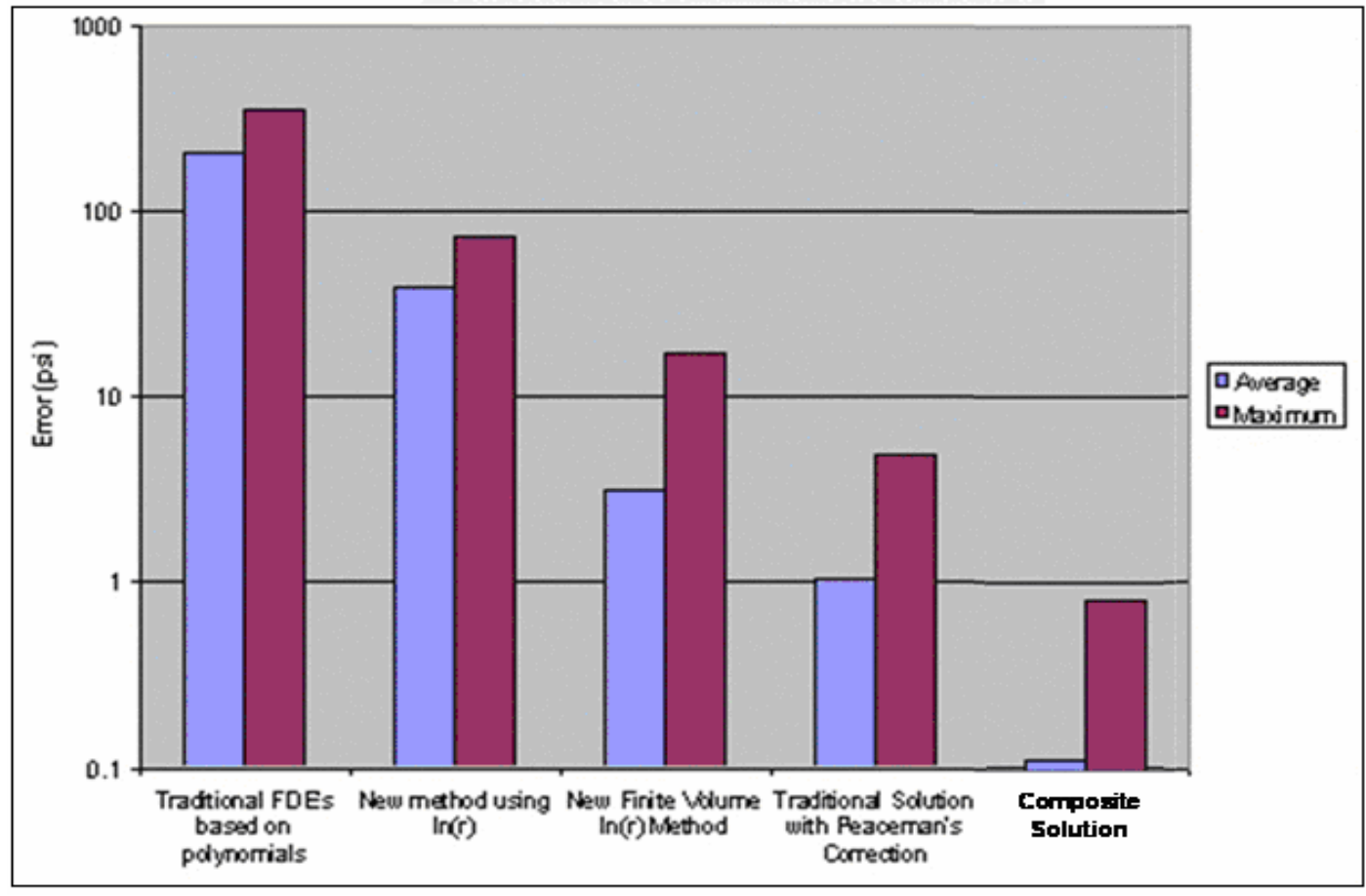

Figure 2. Comparison of Weber's and Composite Solution Results

All results were obtained with a 9 by 18 grid consisting of 100 foot square cells. A sixinch diameter injection well was centered in the center of the right-hand 9 by 9 segment, 
and a production well in the left. Well pressures were \pm 1000 psi. Errors are the differences between the finite-difference results with the analytical solution of MorelSeytoux. As can be seen, the new composite method is nearly an order of magnitude more accurate than both Weber's and Peaceman's results.

Results for this investigation, shown in the subsequent figures, were for a similar reservoir simulated with a 5 by 10 grid of 100 foot square cells. Injection was at $1500 \mathrm{psi}$ and production at -1500 psi. Figure 3 demonstrates how the composite solution can be included only in cells near the wells. Weber found an optimum patch size of only nine cells in each layer, i.e., just the well cells and their immediate neighbors. The composite solution, however, shows no such optimum. The best results are obtained using the composite solution throughout the reservoir. However, using the composite solution in only one cell, the well cell, resulted in results very similar to Peaceman's. In fact, the two solutions are identical, except Peaceman's experimental results included an empirical constant, $\mathrm{c}=0.2$. The analogous composite solution's theoretical constant is $\mathrm{c}=\mathrm{e}^{-\pi / 2}=$ 0.20788 .

Figure 3 shows the results of using a composite solution using an analytical solution from only one well, the closest one. The sum in Equation (16) is omitted. It also shows the "two well" solution which includes the equation (16) sum over "all wells". There are only two in the reservoir. It can be seen that the results are very comparable, but the one well solution is actually slightly better when all the cells in the reservoir include the composite method. 


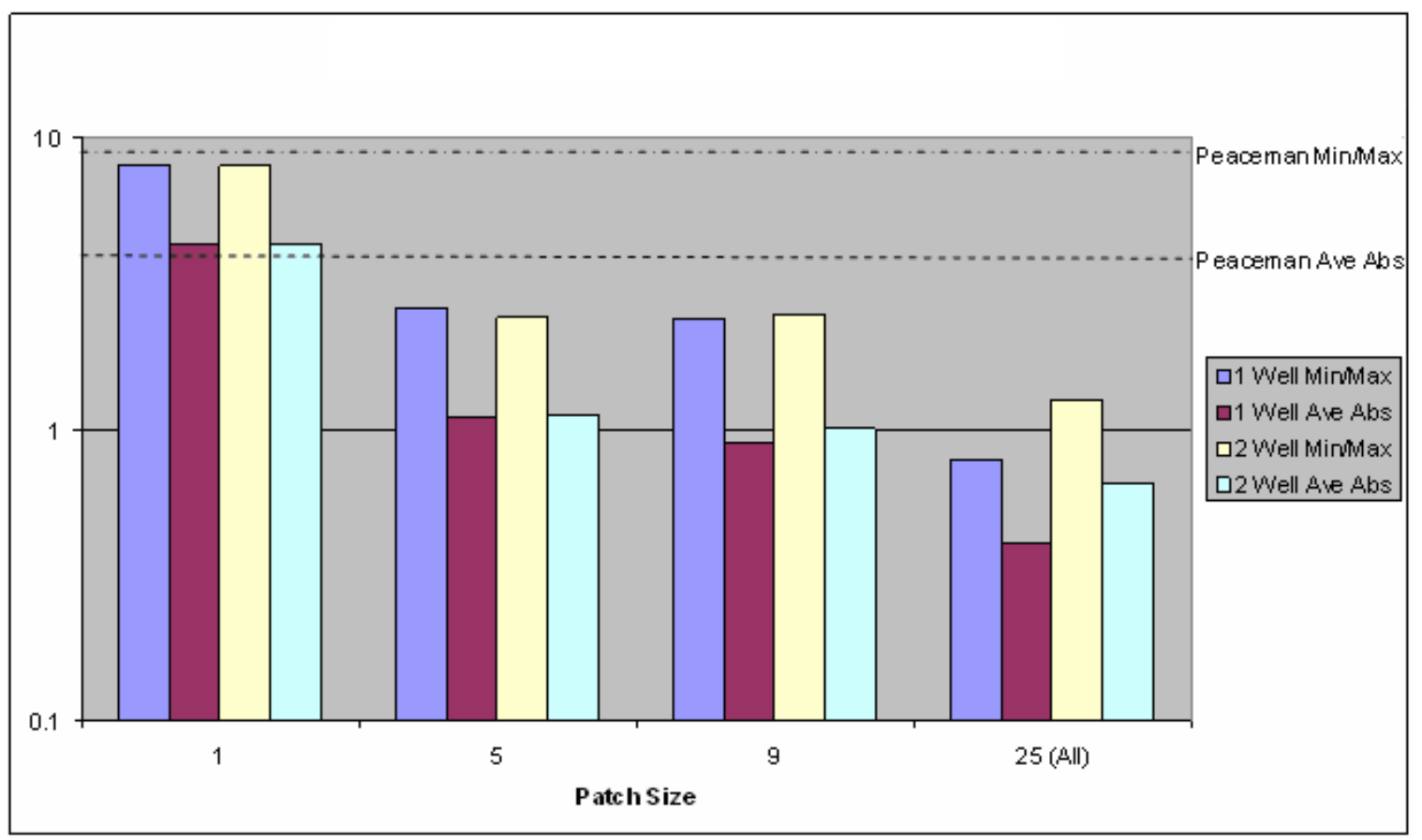

Figure 3. One Well and Two Well results at varying patch sizes

Figure 4 shows that the wells can be can be moved from the cell centers without substantially affecting the error. The wells were moved simultaneously along the reservoir centerline between them maintaining symmetric locations in the two reservoir halves. Well separation distances ranged from $20 \mathrm{ft}$, nearly superimposed, to $980 \mathrm{ft}$, where each well was only $10 \mathrm{ft}$ from the reservoir boundary. The figure shows several things: First it confirms the results of Figure 2, that the "1 well sum" is best when the wells are far from one another and far from the boundaries. However it also shows that as the wells get close to one another, the " 2 well sum" is preferable. Both of the reservoir's wells should be included in the sum of Equation (16). Finally, it shows that when the wells get close to the reservoir boundary, a four well sum is preferable. Two additional, hypothetical, mirror-image wells placed across the near boundaries are included in the sum. 


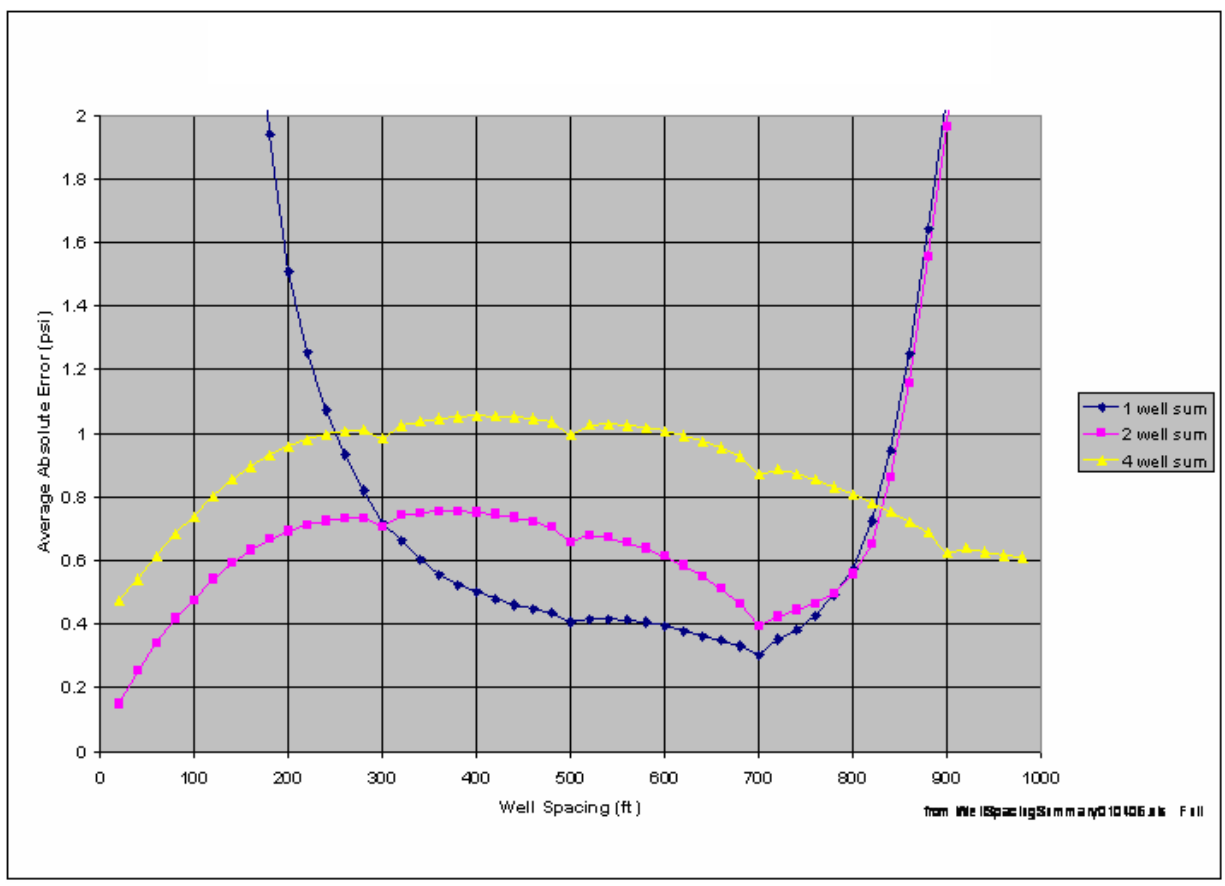

Figure 4. Effect of well location in full grid composite solution

Figure 5 shows similar results when only one-cell patches are used at the wells as the composite solution. As discussed previously, this makes the solution nearly the same as Peaceman's when the wells are in the center of the cells. The figure shows only small variations in the error as the wells are moved along the reservoir centerline between them, as in Figure 3. The over-sized data points are those where the wells are centered in the cells, and correspond to the Peaceman solution. Peaceman pointed out that the well pressure changes little as the well moves across an isolated cell. However, the surrounding cell pressures do. That is, Morel-Seytoux's analytical solution changes. Hence Peaceman's average reservoir pressure error rises rapidly as the wells deviate from the cell center. 


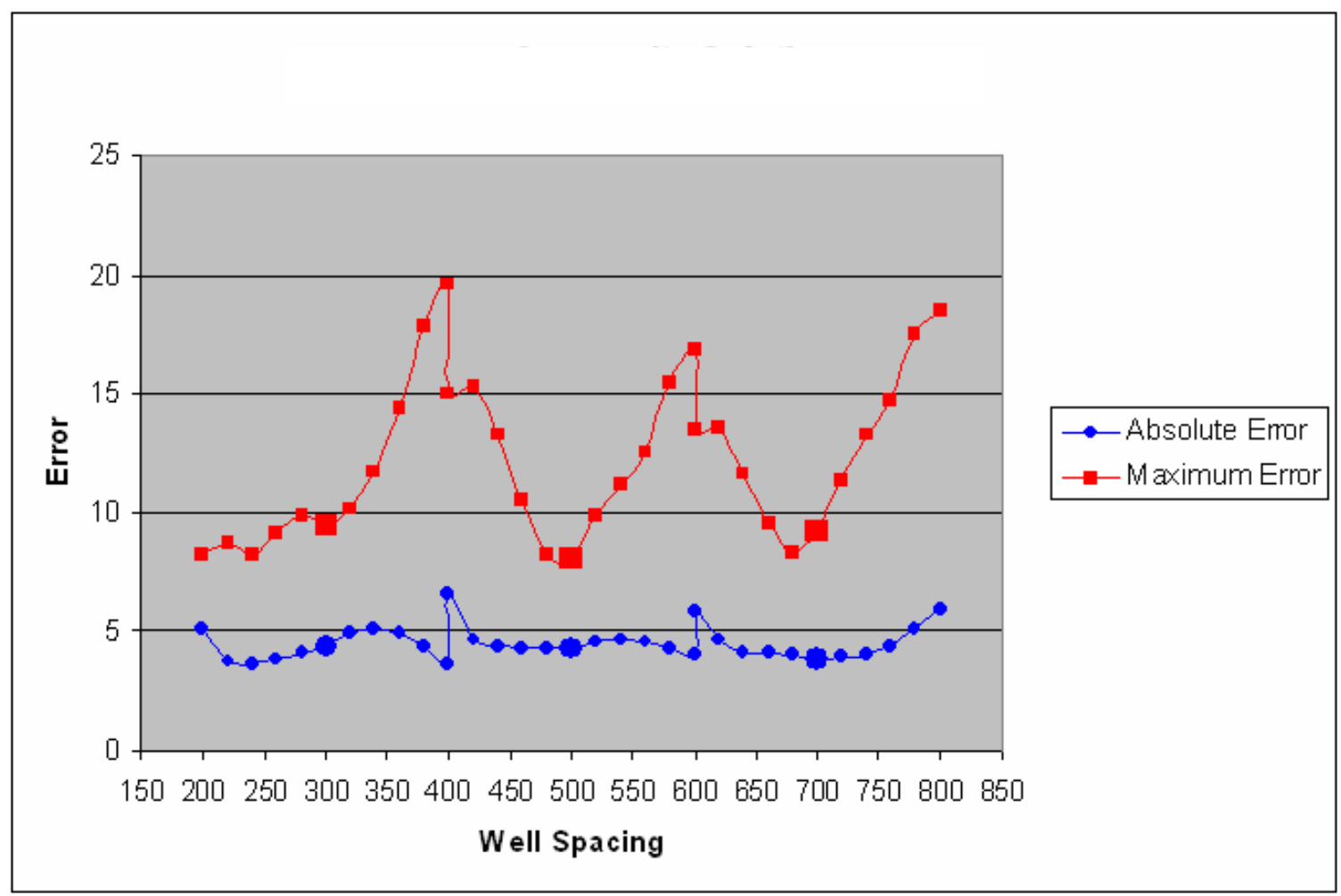

Figure 5. Results of well placement for one-cell composite solution

Figure 6 shows the maximum and absolute errors in the pressure for Peaceman's solution and the composite solution as the well is moved horizontally through the well cell. A well spacing of 500 corresponds to the wells being centered in the cells, and 400 and 600 being the wells on the cell boundaries. 


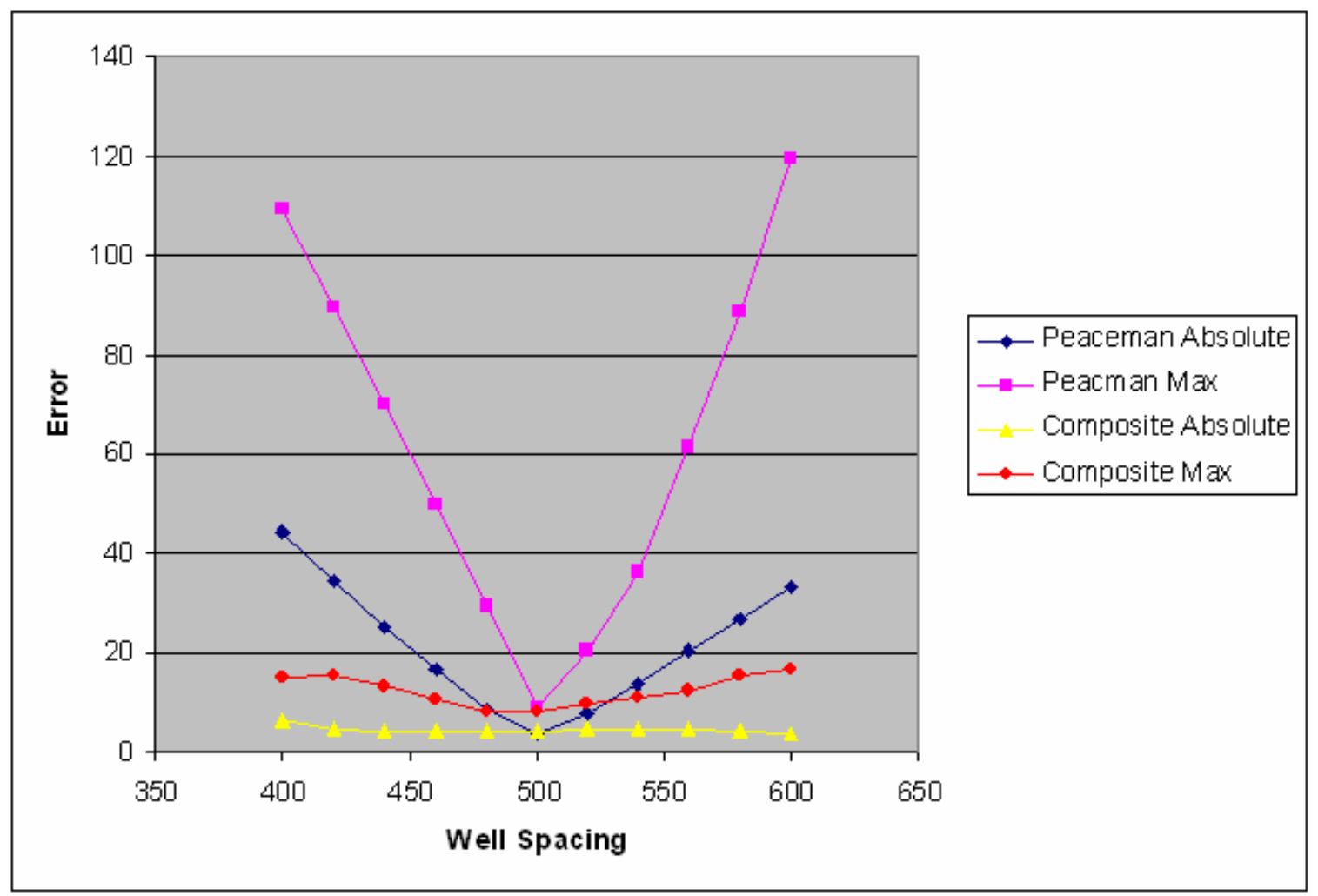

Figure 6. Errors of Peaceman and Composite solutions for offcenter wells 


\section{Chapter 5}

\section{Conclusion}

Incorporation of approximate analytical expressions for the pressure into the reservoir simulator's finite difference equations improves the accuracy of the solutions, especially around the wells. Combining analytical solutions with traditional finite difference equations so that the finite difference component is the error in the analytical solution provides even greater accuracy. These new methods allow the well to be placed anywhere in the cell and may be able to accurately describe wells of arbitrarily complex geometry. 


\section{Nomenclature}

$\begin{array}{lll}\mathrm{P} & = & \text { pressure } \\ \mathrm{r} & = & \text { radial distance from the well } \\ \mathrm{r}_{\mathrm{w}} & = & \text { well radius } \\ \mathrm{Q} & = & \text { well rate } \\ \mathrm{K} & = & \text { permeability } \\ \mathrm{h} & = & \text { reservoir thickness } \\ \mathrm{x}, \mathrm{y} & = & \text { Cartesian coordinate distances }\end{array}$

Greek

$$
\begin{array}{lll}
\mu & = & \text { fluid viscosity } \\
\triangle \mathrm{X} & = & \text { grid spacing distance } \\
\Sigma & = & \text { Sum for all wells } \\
\Omega & = & \text { Solid Angle }
\end{array}
$$

$\underline{\text { Subscripts }}$

$\begin{array}{ll}\text { well } & =\text { pertaining to the well } \\ \text { cell } & =\text { pertaining to the finite difference } \\ \mathrm{i} & =\text { grid point index number } \\ \mathrm{n} & =\text { well index number } \\ \mathrm{a} & =\text { analytical solution } \\ \mathrm{f} & =\end{array}$




\section{References}

1. PEACEMAN, D.W., Interpretation of Well-Block Pressures in Numerical Reservoir Simulation, Society of Petroleum Engineers Journal, June 1978, pp 183-194.

2. PEACEMAN, D.W., Interpretation of Wellblock Pressures in Numerical Reservoir Simulation: Part 3-OffCenter and Multiple Wells Within a Wellblock. SPE Reservoir Engineering, May 1990, pp227-232.

3. KUNIANSKY, J. and Hillestad, J.G., Reervoir Simulation Using Bottomhole Pressure Boundayr Conditgions, SPEJ, December 1980, pp473-486.

4. WILLIAMSON, A.S. and CHAPELLEAR, J.E., Representing Wells in Numerical Reservoir Simulation: Part 1-Theory, SPEJ, June 1981, pp323-338.

5. WILLIAMSON, A.S. and CHAPELLEAR, J.E., Representing Wells in Numerical Reservoir Simulation: Part 2-Implementation, SPEJ, August 1981, pp339-344.

6. ABOU-KASSAM, J.H., and AZIZ, K., Analyical well models for Reservoir Simulation, SPEJ, August 1985, pp573-579.

7. DING, Y, LEMONNIER, P.A., ESTEBENET, T., and MAGRAS, J-F., Control-Volume Method for Simulation in the Well Vicinity for Arbitrary Well Configurations, SPEJ. March 2000, pp 118-125.

8. WEBER, D.B., HALES, H.B., and BAXTER, L.L., A New Method of Formulating Finite Difference Equations-Some Reservoir Simulation Examples, Paper 2004-170 presented at the Petroleum Society's $5^{\text {th }}$ Canadian International Petroleum Conference, Calgary, Alberta Canada, June 8-10, 2004.

9. TAYLOR, B., Methodus Incrementorrum Direcdta et Inversa, Londini (bg): Innys, London, England, 1715.

10. MOREL-SEYTOUX, H. J., Unit Mobility Ratio Displacement Calculations for Pattern Floods in Homogeneous Medium, SPEJ, September, 1966, pp 217-246.

11. CHRISTIE, M.A., Flow in Porous Media-scale up of Multiphase Flow, Current Opinion in Colloid \& Interface Scince, 6 (3): 236-241 Jun 2001.

12. WARREN, J.E. and PRICE, H.S., Flow in heterogeneous Porous Media, SPEJ, September 1961, pp222.

13. MUSKAT, M. Flow of Homogeneous Fluids Through Porous Media, McGraw-Hill Book Co., New York City (1937).

14. CARDWELL, W.T. jr. and PARSONS, R.L., Average Permeabilities of Heterogeneous Oil Sands, Trans., AIME 1945 pp34

15. LI, D., BECKNER, B., and KUMAR, A., A New Efficient Averaging Technique for Scaleup of Multimillion-Cell Geologic Models, 1999 SPE Annual Technical Conference and Exhibition, Houston, 3-6 Oct.

16. BEGG, S.H., CARTER, R.R, and DRANFIELD, P., Assigning Effective Values to Simulator Gridblock Parameters for Heterogeneous Reservoirs, SPERE, November 1989, pp455.

17. STONE, H.L., Rigorous Black-Oil Pseudofunctions, SPE paper 21207 presented at the 1991 SPE Symposium on Reservoir Simulation, Anaheim, California, 17-20 February.

18. PICKUP, G.E., et al. A Method for Calculating Permeability Tensors Using Perturbed Boundary Conditions, Proc., Third European Conference on the Mathematics of Oil Recovery, Christie, M.A., et al. (eds.), Delft U. Press, Delft, The Netherlands, 1992, p225.

19. KING, M.J., BURN, K.S., WANG, P.,MURALIDHARN, V., ALVARADO, F., and MA, X., Optimal Coarsening of 3D Reservoir Models for Flow Simulation, SPE paper 95759 presented at the 2005 SPE Annual technical conference and Exhibition, Dallas, Texas, 9-12 October 2005. 
20. KYTE, J.R., and BERRY, D.W., New Pseudo Functions to Control Numerical Dispersion, SPEJ, August 1975, pp269.

21. STONE, H.L., Rigorous Black-Oil Pseudofunctions, SPE paper 21207 presented at the 1991 SPE Symposium on Reservoir Simulation, Anaheim, California, 17-20 February.

22. HARDY, B.A., HALES, H.B, BAXTER, L.L., A New Method for the Rapid Calculation of Finely Gridded Reservoir simulation Pressures, CIPC paper 2005-112 presented at the Petroleum Society's $6^{\text {th }}$ Canadian International Petroleum Confernce, Calgary, Alberta, Canada, June 7-9, 2005.

23. VAN POOLEN, H.K., BREITENBACH, E.A., THURNAU, D.H., Treatment of Individual Wells and Grids in Reservoir Modeling, SPEJ, December, 1968, pp 3414-346, Trans., AIME, 243 
APPENDIXES 


\section{APPENDIX A WORK NOT INCLUDED IN THESIS BODY}

The original objective of this thesis was to extend the work of Hardy ${ }^{22}$ so that it could be used in commercial and research simulators 1) by extending the work to more general well geometries, and 2) by extending the work to heterogeneous reservoirs. Some important results were obtained in extending the work to general well geometries and they were described in the main body of the thesis. Although the majority of the time for the research was spent investigating heterogeneous reservoirs, it was not as successful. It was found that the level of accuracy was not sufficient to exceed that of existing methods. For that reason, this appendix contains the work that was done in extending the Hardy's work with homogeneous reservoirs to handle heterogeneous reservoirs.

\section{Introduction}

Reservoir simulation is an important tool in the petroleum industry. Simulators are used to predict how much a reservoir will produce and how quickly. Despite constant improvement of computing resources, reservoir simulations are limited by the computer power available. Current technology allows simulations involving 10,000 - 100,000 cells to be run in a reasonable amount of time ${ }^{11}$. This causes a problem because the model for the geological properties is generally on a scale of a million cells. The geological model must be coarsened in order to be used in a reservoir simulation. This is done by a process known as "upscaling." 
Upscaling is done by taking the fine geological model and converting it into course grid that has equivalent reservoir rock properties. This coarser grid can then be used in a simulator. The problem with upscaling is trying to maintain an accurate description of the reservoir properties that are important to fluid flow. Upscaling is basically done in two steps. First, the reservoir permeabilites and other grid properties are adjusted to provide the same reservoir pressures and single phase reservoir flows as exist with the coarse grid. Second, relative permeabilites are adjusted to compensate for changes to multiphase flow caused by the grid coarsening.

The goal of single-phase upscaling is to preserve the gross flow features in the simulation. This is done by calculating effective permeabilities for the coarse grid blocks that result in the same flow through the cell as compared to the finer blocks that make up the coarse block.

The simplest and quickest way to handle single-phase upscaling is averaging the permeabilities in the fine cells to get an equivalent permeability for the coarse cell. The difficulty with these methods is deciding how to average the permeabilities. Averaging techniques give good results when the permeability is randomly distributed through the medium. Warren and Price ${ }^{12}$ have shown that in a randomly generated 3D permeable medium the effective permeability is equal to the geometric mean of the individual permeabilities. Unfortunately, permeabilities in actual reservoirs are not randomly distributed. Most reservoirs will have layers with varying permeability. The effective permeability of a medium with homogeneous layers is the arithmetic mean if the flow is parallel to the layering and the harmonic mean if flows are perpendicular to the layering ${ }^{13}$. It has also been proven that when the layers are heterogeneous the arithmetic and 
harmonic means provide upper and lower bound for the effective permeability ${ }^{14}$. The problem then becomes a question of how to combine the averages to get the effective permeability that will preserve the fine-scale properties.

Averaging techniques are generally the fastest but least accurate. For large reservoirs it may not be feasible to use the more accurate techniques. Li et al. ${ }^{15}$ have developed an averaging method that attempts to preserve the accuracy of simulation methods while retaining the speed of averaging methods. The upper and lower bounds of the effective permeability are first calculated and a new correlation, scaling and rotation technique is used to estimate the effective permeability in the coarse grid blocks. Full tensor permeabilities result. That is, the velocity vector in the coarse grid cell is not parallel to the pressure gradient. For example, a pressure gradient in the $\mathrm{x}$-direction results in flow not only in the x-direction but also in the $y$ - and z-directions. The method was tested on five actual reservoirs and was shown to be a good upscaling technique that has the advantage of being able to handle irregular geometries.

Pressure-solver methods are more accurate than averaging techniques, but a solution of the fine-scale pressure equation is necessary and hence they are more difficult to obtain. One of the first single-phase, pressure-solver upscaling methods was developed by Begg et al ${ }^{16}$ In this method an effective permeability is calculated that will give the same flow as the fine-grid by solving the pressure equation with no flow boundary conditions on the cell edges parallel to flow. The chosen boundary conditions and their accompanying assumptions will influence the effective permeability calculated. The main advantage of this technique is that the no-flow boundary conditions give a diagonal 
tensor that leads to much faster solutions than full tensor permeabilities. Even though the fine detail of the reservoir is lost, the gross features of single phase flow are preserved.

Full-tensor effective permeabilities are obtained when periodic boundary conditions are used. Periodic boundary conditions surround the cell by copies of itself so that flow passing out of the cell will enter the cell through the opposite face. Periodic boundary conditions generally give more accurate results than no-flow boundary conditions. Durlofsky ${ }^{17}$ provides a summary of scale-up using tensor permeabilities and favors using periodic boundary conditions. Pickup ${ }^{18}$ tested the accuracy and robustness of several boundary conditions when calculating effective permeabilities and also found periodic boundaries to be the best.

Recently a new approach to single-phase upscaling has been developed by King et al. ${ }^{19}$ where the focus is on optimizing how the reservoir grid is coarsened. The goal is to minimize the variance of the permeability in each coarse grid block. This results in grid blocks of varying shape and size, but optimally preserves the fine grid properties. The method does not require a fine-grid pressure solution and also reduces computational cost by being based on sequential recursive coarsening which scales with the number of grid blocks rather than sequential refinement which scales with the square of the number of grid blocks. Along with an accurate measure of reservoir heterogeneity, this provides a method that is both fast and accurate. However, highly unstructured grids result, requiring increased computer time for their solutions.

Mutli-phase upscaling presents a much greater challenge than single-phase. The absolute permeability is the most important parameter in single-phase, but multi-phase has to also adjust phase flow through connections of the coarse grid. The best approach to 
multiphase upscaling is still debated, but the most widely used technique employs pseudo relative permeabilities. The process involves replacing the original, fine grid, saturation dependent functions by ones that will represent the system on a coarser scale

The most common procedure to calculate such pseudo permeability curves was developed in 1975 by Kyte and Berry ${ }^{20}$. In their method the average pressures, total flow rates, and phase potentials for each coarse block are estimated from the fine-grid values. This is then substituted into the course-grid Darcy equation to calculate what the pseudo relative permeabilities must be in order to produce the fine-grid flows. While it is the most common method, it does not give good results in strongly heterogeneous media and negative or infinite values of the relative permeability can occur.

Stone ${ }^{21}$ found a way to avoid calculating phase potential on the coarser grid and the problems associated with this in the Kyte and Berry method. Fractional flow formulations are used instead of Darcy's Law. This eliminates the use of phase potentials and the total mobility is used to calculate the pseudo-relative permeability. This can be accomplished only if capillary pressure and gravity are neglected, which puts limitations on the use of this method.

\section{Outline of Objectives and Significance of Work}

The objective of this work was to generalize the new pressure solution method developed at BYU by Ben Hardy ${ }^{22}$ so that it might readily be incorporated into both commercial and research reservoir simulators. The process was divided into three parts:

The first step was to investigate upscaling methods and choose the method to be used in Hardy's pressure solution algorithm. The literature was reviewed, as discussed previously, and it was decided to use averages for the upscaled permeabilities. Averaging 
the fine grid permeabilities to get the effective coarse grid permeabilities is fast and does not result in a distorted coarse grid. It was felt that average permeabilities may be adequate for the pressure solution algorithm whereas some investigators have found them inadequate for upscaled simulations and have resorted to the more complex and time consuming techniques. Averages may be adequate because the coarse grid solutions obtained with the upscaled permeabilities are intermediate solutions in the Hardy algorithm which are used to obtain a fine grid solution, not the final solution. Fine scale flows do not have to be represented by coarse grid permeabilities. Arithmetic, harmonic, and geometric averages were used as well as combinations of these averages. Several synthetic heterogeneous permeability data sets were generated combining varying amounts of homogeneous layering with random permeability variations. Hardy's pressure solution method would then be used to find the pressure in these systems. These solutions were compared with the exact solution for these systems by removing the Hardy fixed points and allowing the solution to continue to iterate to convergence. Results for the various averages were compared.

The work of Weber et al. ${ }^{8}$ showed that the error in the pressure equation could be reduced by five orders of magnitude by incorporating the singularities in the reservoir pressures, resulting from the wells, into finite difference equations. The second part of this thesis was to extend Weber's work to apply to wells of arbitrary geometry. Instead of using traditional polynomial expressions, Weber used $\ln (\mathrm{r})$ or $1 / \mathrm{r}$ functions in the derivation of his finite difference equations to find the pressures resulting from straight line wells and point source wells respectively where $r$ is the distance from the well. Weber only investigated straight-line and point-source wells. Weber's research was also 
done on homogeneous systems, so it is necessary to see what happens when it is applied to a heterogeneous system.

The tremendous accuracy of Weber's solution is interesting, but of little practical use for reservoir simulation because fine grids are required not only to insure the accuracy of the finite difference solutions, but also to incorporate the details geologic description such as variations in permeability and porosity. The work of Hardy et al. was to use Weber's highly accurate course grid pressure solutions to greatly increase the speed at which the fine-grid pressure could be calculated. This is done by solving for the pressure on a finer grid with the coarse-grid solution nested in as fixed points. This allowed the fine grid solution to be found very quickly and efficiently.

\section{Results}

Averaging is the quickest and simplest way to upscale permeabilities in a reservoir simulator. It is also the method that could be best implemented into Hardy's nested-grid, pressure-solver algorithm. For these reasons averaging techniques were used to investigate how heterogeneous media affected the accuracy of Hardy's method. The model reservoir used by Hardy is shown in Figure A-1.

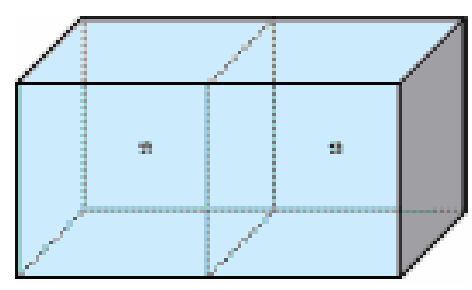

Figure A-1. Model Reservoir with point source wells 
It consists of a rectangular reservoir with an injection well centered in one half and a production well centered in the other. Weber's $1 / \mathrm{r}$ solution is used for point source wells.

The difficulty that lies in using averaging techniques is deciding what type of average to use. The choice between geometric, harmonic, and arithmetic means depends on the nature of the heterogeneous media. If the permeabilities are random in all directions then the geometric mean is the best representation. If the media is made of homogeneous layers then the harmonic mean is the best if flow is perpendicular to the layers. If the flow is parallel to the layers then the arithmetic mean should be used. Initially, random distributions were studied and hence, the geometric mean was used. Hardy's Fortran algorithm consists of two parts. The first part solved the pressure on a coarse grid of desired grid dimensions ranging from $5 \times 5 \times 10$ to $65 \times 65 \times 130$. These pressures would then be read into one of seven other programs that would solve for the pressure on a fine grid using some of the coarse grid pressures as nested points. The choice of the second program depended on the number of nested grid points that was desired ranging from 18 to 31250 fixed points.

Adding heterogeneity to the system was accomplished by first generating an array of $\log$ normally distributed random permeabilties. These could then be assigned to grid blocks in both the coarse and fine grid programs. The coarse grid program had to be modified to correspond to a specific size of fine grid. Coarse grid blocks were made up of 27 fine grid blocks. For this reason the coarse grid block dimensions had to be exactly three times the size of the fine grid. For example, a coarse grid with $5 \times 5 \times 10$ blocks corresponds to a fine grid of $15 \times 15 \times 30$ blocks. 
Once the permeabilities were available to both programs the upscaling was accomplished in the coarse grid program. This was done by averaging the permeabilities in the 27 fine grid blocks that corresponded to one coarse grid block to get its upscaled permeability. The coarse grid solution could then be solved and the desired number of nested grid points could be read into the fine grid solver and the fine grid solution could be calculated. The error was calculated by comparing the coarse grid solution with the fine grid solution.

As previously mentioned the difficulty in using averaging techniques in upscaling is deciding on how to average the permeabilities. Since the permeabilities were randomly distributed a geometric mean should give the best results. This was accomplished by taking the geometric mean of the 27 fine grid blocks that made up one coarse grid block to get its permeability. Now, since the solver used finite difference equations to solve for the pressure it is not the grid block permeability that is needed, but a linking permeability between two grid blocks. This was accomplished by harmonically averaging the two grid block permeabilities to arrive at a linking permeability. However, this was probably not the best way to arrive at a linking permeability because it involved all 54 of the fine grid permeabilities that make up the two cells. A better approach would be to get an upscaled coarse grid permeability using only the fine grid permeabilities that lie between the two cell centers. This would mean that only the cells that are in the shaded area of Figure A-2 would be used. 


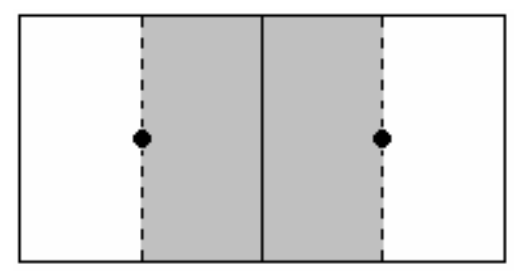

Figure A-2. Example of area used to calculate the linking permeability between two cells

The geometric mean calculated in this fashion involved the 36 fine grid blocks that fall in the shaded area. The average is weighted so that the nine blocks that lie along the center of either cell only contribute half as much since only half of the block lies in the shaded area.

A combination of the harmonic and arithmetic mean was also considered. Again, it was calculated using the fine grid permeabilities that lie between two grid blocks to get a linking permeability. A harmonic mean was taken in the horizontal direction for each horizontal row of cells, and then the nine rows were averaged arithmetically to get the upscaled linking permeability.

Three random permeability sets were produced using a log normal distribution with a mean of 1.0 and a standard deviation of 0.2 to test the accuracy of solutions produced with the various averaging techniques. The tests were run using a grid of $15 \times 15 \times 30$ grid blocks with a corresponding $5 \times 5 \times 10$ coarse grid. The injection and production wells were at pressures of 1500 and -1500 psi respectively. Figure A-3 shows the average absolute error in the pressure for the three data sets using the three methods of averaging: geometric mean including all fine grid cells, combination of harmonic and arithmetic mean using only fine grid cells in between, and geometric mean using only fine grid cells in between. 


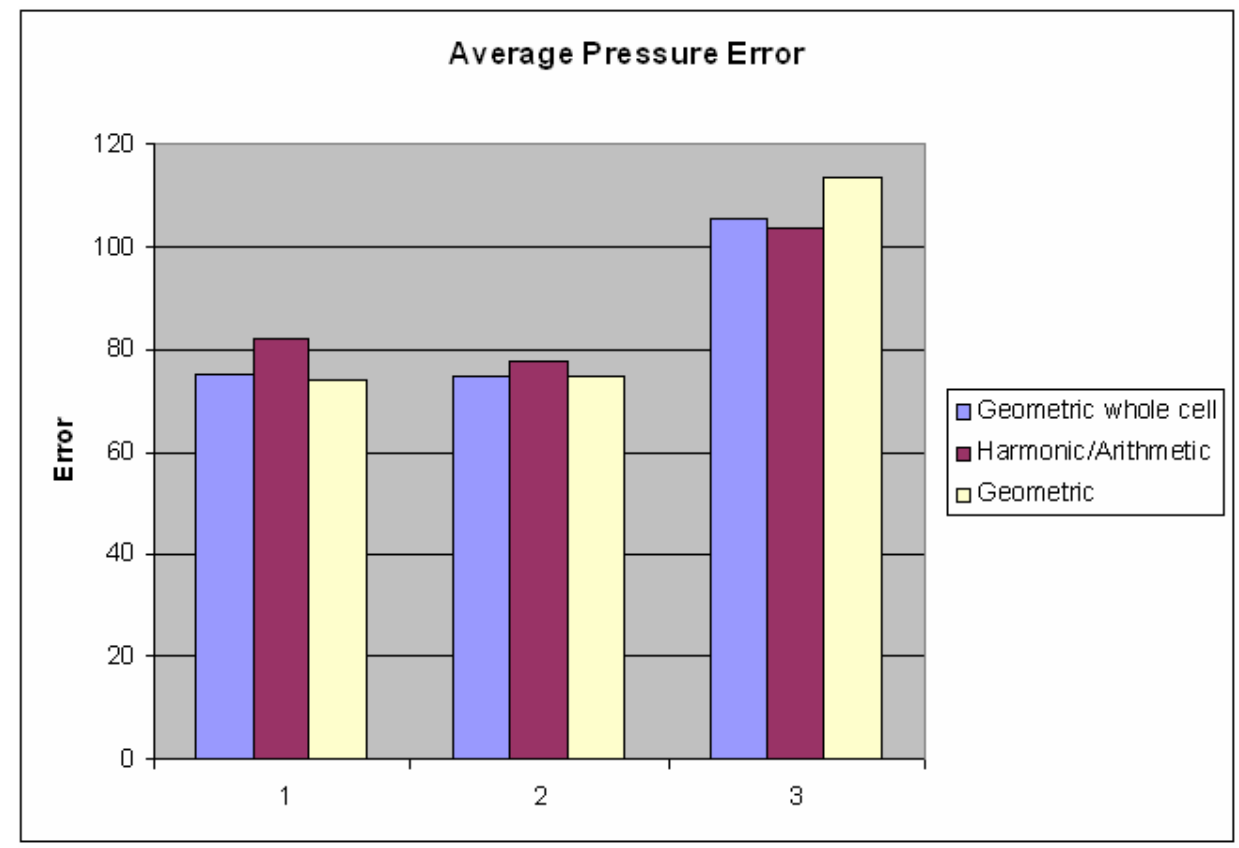

Figure A-3. Upscaling results

An increase in the error was expected when moving to heterogeneous reservoirs, but these results were much greater than anticipated. The homogeneous case gave an error on the order of 1 psi.

Upon closer inspection of the results it was seen that the error was affected almost solely by the permeabilities in the two well cells. If the upscaled coarse grid permeability did not closely match the fine grid well cell permeability then large errors occurred. This was confirmed by running the same data sets over, except with the well cell permeabilities equal to each other. This meant that the permeabilities of the coarse grid well cell and the corresponding 27 fine grid cells at both wells were equal. The results were that the error was on the order of $1 \mathrm{psi}$, which is about the same as the homogeneous result. By changing the permeabilities around the well to be homogeneous, upscaling was 
not necessary at the wells and the upscaling method used elsewhere became unimportant since the upscaling around the wells had the greatest impact on the errors.

This meant that the area around the wells had to somehow be treated differently than the rest of the reservoir. To this end coefficients were derived to better evaluate the effective peremeabilities that result from the upscaling. The derivation is as follows. If flow emerges from a spherical well and flows in the radial direction only (i.e. 1-D spherical flow) then

$$
Q=4 \pi r^{2} \frac{k(r)}{\mu} \frac{d p}{d r}
$$

If $\mathrm{k}$ is piece-wise constant, i.e. constant within each cell, then within any cell

$$
p-p_{i}=\frac{Q \mu}{4 \pi k_{i+}}\left(\frac{1}{r_{i}}-\frac{1}{r}\right)
$$

Where $r_{i}$ is the radius of the cell edge closest to the well and $p_{i}$ is the pressure there. In the well cell $r_{i}$ equals the well radius. The pressure difference between adjacent finite difference grid points is therefore given by:

$$
\begin{aligned}
p_{i+1}-p_{i} & =\left(p_{i+1}-p_{i+1 / 2}\right)+\left(p_{i+1 / 2}-p_{i}\right) \\
= & \frac{Q \mu}{4 \pi}\left(\frac{1}{k_{i+1}}\left|\frac{1}{r_{i+1}}-\frac{1}{r_{i+1 / 2}}\right|+\frac{1}{k_{i}}\left|\frac{1}{r_{i}}-\frac{1}{r_{i+1 / 2}}\right|\right)
\end{aligned}
$$


The flow passing through the $i+1 / 2$ cell face is given by

$$
Q_{i+1 / 2}=\frac{Q}{4 \pi} \Omega
$$

Where $\Omega$ us the solid angle swept by the $i+1 / 2$ cell face relative to the well.

Now the effective permeability is given by

$$
k_{i+}=\frac{Q_{i+1 / 2} \mu \Delta x}{\left(p_{i+1}-p_{i}\right) A}
$$

Which for equal grid spacing becomes

$$
k_{i+}=\frac{\Omega}{\Delta x\left(\frac{1}{k_{i+1}}\left|\frac{1}{r_{i+1}}-\frac{1}{r_{i+1 / 2}}\right|+\frac{1}{k_{i}}\left|\frac{1}{r_{i}}-\frac{1}{r_{i+1 / 2}}\right|\right)}
$$

If we want to upscale and get the effective permeability between cell $\mathrm{i}$ and cell $\mathrm{i}+\mathrm{n}$, the formula is similar:

$$
k_{i+}=\frac{\Omega}{\Delta x\left(\frac{1}{k_{i+n}}\left|\frac{1}{r_{i+n}}-\frac{1}{r_{i+n+1 / 2}}\right|+\frac{1}{k_{i}}\left|\frac{1}{r_{i}}-\frac{1}{r_{i+1 / 2}}\right|+\sum_{j=i+1}^{n} \frac{1}{k_{j}}\left|\frac{1}{r_{j}}-\frac{1}{r_{j-1}}\right|\right)}
$$


Where $\Omega$ is now the solid angle swept by the coarse grid cell face between $\mathrm{i}$ and $\mathrm{i}+\mathrm{n}$, and $\Delta \mathrm{x}$ is the grid spacing of the coarse grid.

This work was intended to investigate both point source and straight line well, and at this time it was decided to investigate straight line wells first since they are easier than point source wells. For this reason these new coefficients were not implemented into Hardy's Fortran codes, but similar $\ln (\mathrm{r})$ coefficients were used in Excel files that ran similar calculations for straight line wells. Weber's $\ln (r)$ solution was used for this new well geometry, rather than the $1 / \mathrm{r}$ solution used for point source wells. The derivation of the coefficients used for straight line wells is identical to the above derivation for point source wells except that equation (A-2) becomes

$$
p-p_{i}=\frac{Q \mu}{2 \pi k_{i+}}\left(\ln \left(r_{i}\right)-\ln (r)\right)
$$

Which results in equation (A-6) becoming

$$
k_{i+}=\frac{\Omega}{\Delta x\left(\frac{1}{k_{i+1}}\left|\ln \left(r_{i+1}\right)-\ln \left(r_{i+1 / 2}\right)\right|+\frac{1}{k_{i}}\left|\ln \left(r_{i}\right)-\ln \left(r_{i+1 / 2}\right)\right|\right)}
$$

and equation (A-7) becoming

$$
k_{i+}=\frac{\Omega}{\Delta x\left(\frac{1}{k_{i+n}}\left|\ln \left(r_{i+n}\right)-\ln \left(r_{i+n+1 / 2}\right)\right|+\frac{1}{k_{i}}\left|\ln \left(r_{i}\right)-\ln \left(r_{i+1 / 2}\right)\right|+\sum_{j=i+1}^{n} \frac{1}{k_{j}}\left|\ln \left(r_{j}\right)-\ln \left(r_{j-1}\right)\right|\right)}
$$


Straight line wells that penetrated the entire reservoir were used so that each horizontal layer of the reservoir contains wells as shown in Figure A-4.

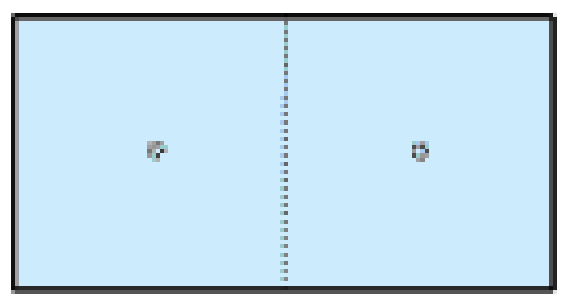

Figure A-4. Horizontal layer of reservoir with fully-penetrating, straight-line wells

The same three permeability data sets that were used to test Hardy's algorithm were used to test the $\ln (\mathrm{r})$ pressure solver. The upscaling was done using the geometric mean of the permeabilities between the two cell centers as discussed previously on page 35. The new coefficients were used to get the linking permeabilities between the well cell and its neighbors. Radial distances were calculated to cell face centers. The results are presented in Figure A-5. 


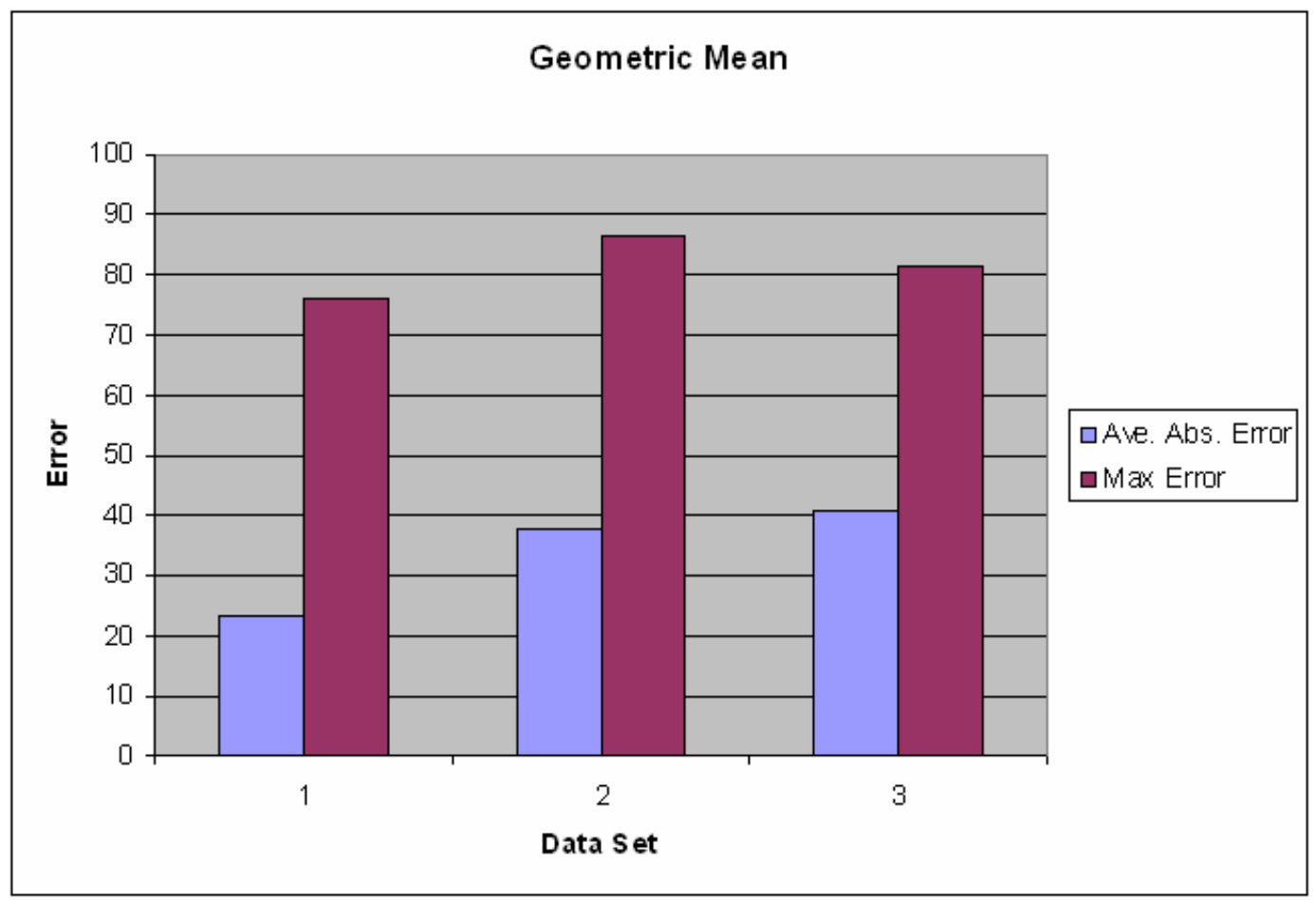

Figure A-5. Upscaling results using the geometric mean

In order to compare this result to the traditional method, the Peaceman correction was implemented into worksheet. The Peaceman correction uses a productivity index to correct the well cell pressure. Peaceman found that the well cell's pressure can be approximated as the actual flowing pressure at a radial distance of $0.2 \Delta x$ from the well.

$$
P_{\text {well }}-P_{\text {cell }}=\frac{1}{2 \pi} \frac{Q \mu}{K h} \ln \left(\frac{0.2 \Delta x}{r}\right)
$$

The upscaling was again done using the geometric mean and the Peaceman correction used in the well cells. The results for the three data sets are shown in Figure A-6. 


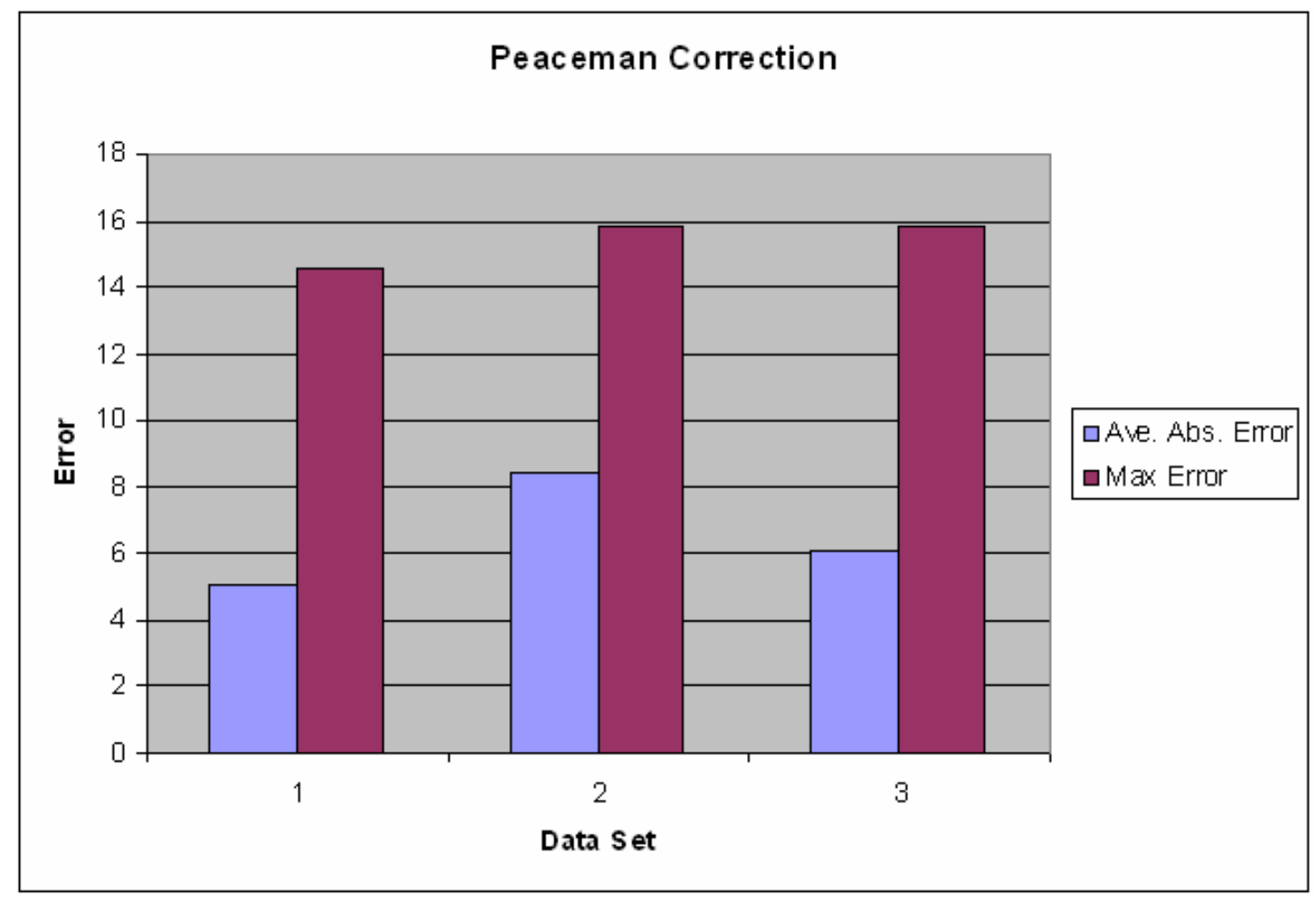

Figure A-6. Upscaling results using Peaceman Correction

From the results it was clear that simply averaging the permeabilities was not a sufficient method to upscale the permeabilities compared to using the Peaceman correction. For this reason a more complicated upscaling method was developed and investigated.

As shown above, equation (A-8) can be used with straight line wells to calculate the pressure drop from one cell to the next if all the values on the right hand side are known. This equation was extended to calculate the pressure drop across multiple cells with differing permeabilities in equation (A-10). However the radius of the cell face centers was used, and the results were not as accurate as desired. Therefore an attempt to integrate over the cell faces to obtain average radius distance was undertaken.

Figure A-7 shows the radial path that flow can take between the well and a neighboring coarse grid cell. The radial lines pass through the corners of the fine grid 
cells. Hence in the areas between the red lines all radii flow through the same cells. One can therefore integrate over all the radii with a slice to obtain the average permeability.

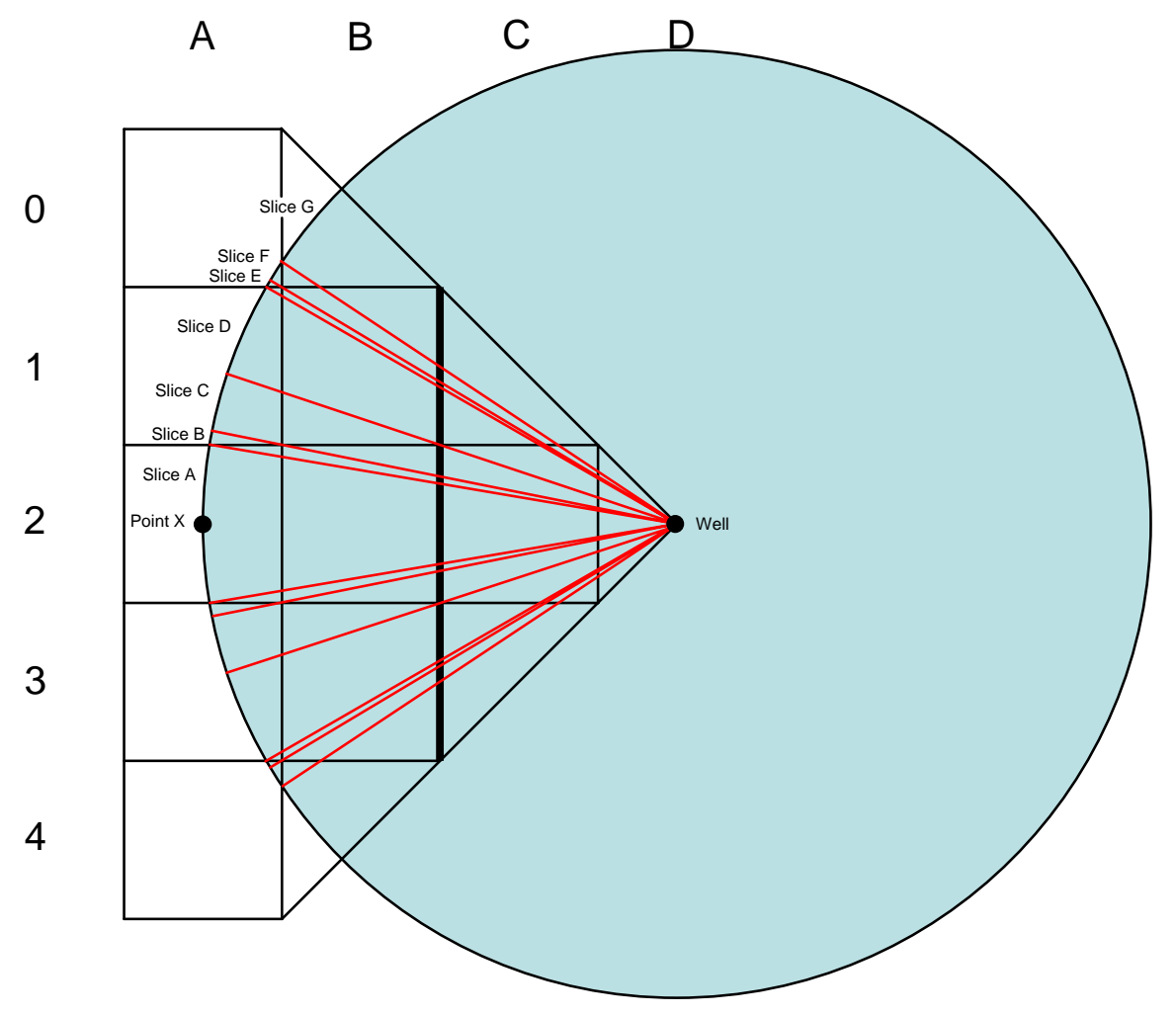

Figure A-7. Illustration of Slices used in Integral Method

Referring to Figure A-7, if the pressure drop between the well and Point X were to be calculated, the formula would look like this:

$$
\Delta p=\frac{Q \mu}{2 \pi}\left(\frac{1}{k_{D 2}} \ln \left(\frac{r_{C D}}{r_{w}}\right)+\frac{1}{k_{C 2}} \ln \left(\frac{r_{B C}}{r_{C D}}\right)+\frac{1}{k_{B 2}} \ln \left(\frac{r_{A B}}{r_{B C}}\right)+\frac{1}{k_{A 2}} \ln \left(\frac{3 \Delta x}{r_{A B}}\right)\right)
$$


where the $k$ 's refer to the permeability in the coarse grid cells and the $r$ 's are the radial distance from the well to the specified face. For example $r_{B C}$ refers to the distance from the well to the face between cells B2 and C2. Equation (A-11) can be combined with (A5) to find the effective permeability along the line between the well and Point $X$. This results in:

$$
k_{\text {eff }}=\frac{\ln \left(3 \Delta x / r_{x}\right)}{\frac{1}{k_{A 2}} \ln (3 \Delta x)-\frac{1}{k_{D 2}} \ln \left(r_{w}\right)+\left(\frac{1}{k_{D 2}}-\frac{1}{k_{D 2}}\right) \ln \left(r_{C D}\right)+\left(\frac{1}{k_{C 2}}-\frac{1}{k_{B 2}}\right) \ln \left(r_{B C}\right)+\left(\frac{1}{k_{B 2}}-\frac{1}{k_{A 2}}\right) \ln \left(r_{A B}\right)}
$$

Since it is possible to find the effective permeability along a line, integration along all the lines in the section can be used to more accurately calculate the effective permeability for an area. To find the upscaled effective linking permeability between the well cell and its neighbor 13 slices were used. Slice A is the central slice and there are slices B through $\mathrm{G}$ for both the top and bottom portions of the area between the cells. The dark vertical line between columns B and C in Figure A-5 shows where the calculated effective permeability applies.

Using a development similar to that used for equation (13) the following equations were derived for each of the slices. In all the equations the radial distances to the faces have been replaced by equivalent expressions in terms of $\Delta x$ and $y$. The terms in the denominator depend on the cells and cell faces that the slice passes through, and since many of the terms are identical they are defined in Table A-1 at the end of this 
section. Also, there are Slices B-G that correspond to the upper portion and similar slices that correspond to the lower portion. The constants presented are for the slices in the upper portion, but can easily be changed to correspond to the lower portion.

For Slice A:

$$
k_{A}=2 \int_{0}^{\frac{1.54 x}{\sqrt{33}}} \frac{\frac{\pi}{2} d y}{A 2 D 2+D 2 C 2+C 2 B 2+B 2 A 2}
$$

For Slice B:

$$
k_{B}=\int_{\frac{1.5 \Delta x}{\sqrt{33}}}^{0.3 \Delta x} \frac{\frac{\pi}{2} d y}{A 1 D 2+D 2 C 2+C 2 B 2+B 2 A 2+A 2 A 1}
$$

For Slice C:

$$
k_{C}=\int_{0.3 \Delta x}^{0.5 \Delta x} \frac{\frac{\pi}{2} d y}{A 1 D 2+D 2 C 2+C 2 B 2+B 2 B 1+B 1 A 1}
$$

For Slice D:

$$
k_{D}=\int_{0.5 \Delta x}^{\frac{\sqrt{3} \Delta x}{2}} \frac{\frac{\pi}{2} d y}{A 1 D 2+D 2 C 2+C 2 C 1+C 1 B 1+B 1 A 1}
$$


For Slice E:

$$
k_{E}=\int_{\frac{\sqrt{3} \Delta x}{2}}^{0.9 \Delta x} \frac{\frac{\pi}{2} d y}{A 0 D 2+D 2 C 2+C 2 C 1+C 1 B 1+B 1 A 1+A 1 A 0}
$$

For Slice F:

$$
k_{F}=\int_{0.9 \Delta x}^{0.3 \sqrt{11} \Delta x} \frac{\frac{\pi}{2} d y}{A 0 D 2+D 2 C 2+C 2 C 1+C 1 B 1+B 1 A 1+B 0 B 1+A 0 B 0}
$$

For Slice G:

$$
k_{G}=\int_{0.3 \sqrt{11} \Delta x}^{1.5 \Delta x} \frac{\frac{\pi}{2} d y}{B 0 D 2+D 2 C 2+C 2 C 1+C 1 B 1+B 0 B 1}
$$

The integrals in equations (A-14)-(A-20) cannot be integrated analytically so Simpson's rule using 100 steps per integral was used to integrate them numerically. Once the effective permeability has been calculated for each slice they can be summed to get the upscaled effective linking permeability.

This integral method is used to find the effective linking permeability between the well cell and its four neighbors only. The geometric mean is used as described on page 35 to find linking permeabilities between all other cells. The hope was that the use of a more 
accurate method of the upscaling around the well would increase the accuracy of the solution. The results are shown in Figure A-8.

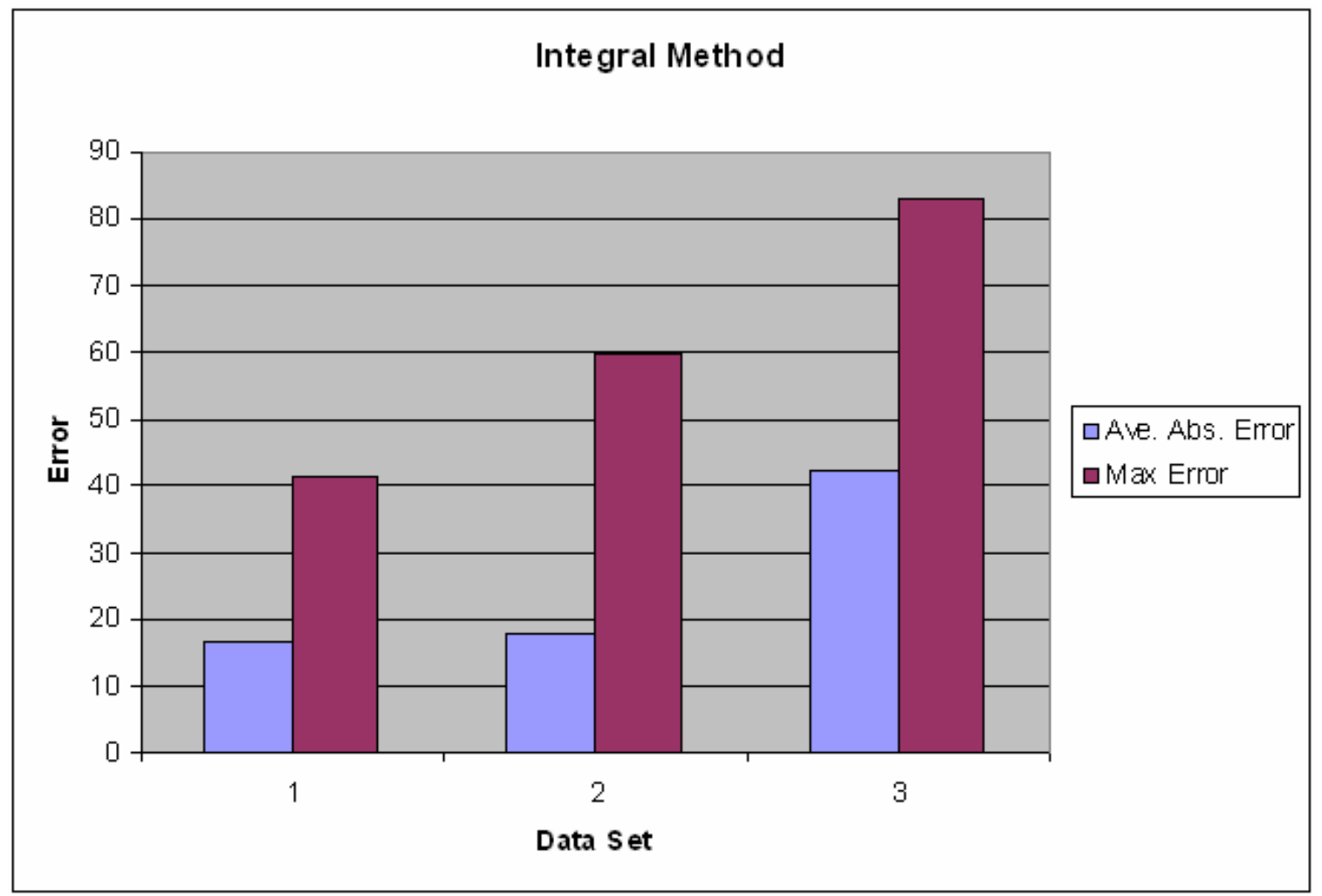

Figure A-8. Upscaling results using the Integral Method

It was found that the accuracy was indeed increased over using only the geometric mean.However, the improvement was disappointing in that it did not show an improvement over using the Peaceman correction. The results comparing the Peaceman correction, the geometric mean, and the Integral method are shown in Figure A-9.

Unfortunately the added complexity of the integral method did not produce the desired level of accuracy. However, the work in the main body of the thesis grew out of this pursuit to improve upscaling around the well. 


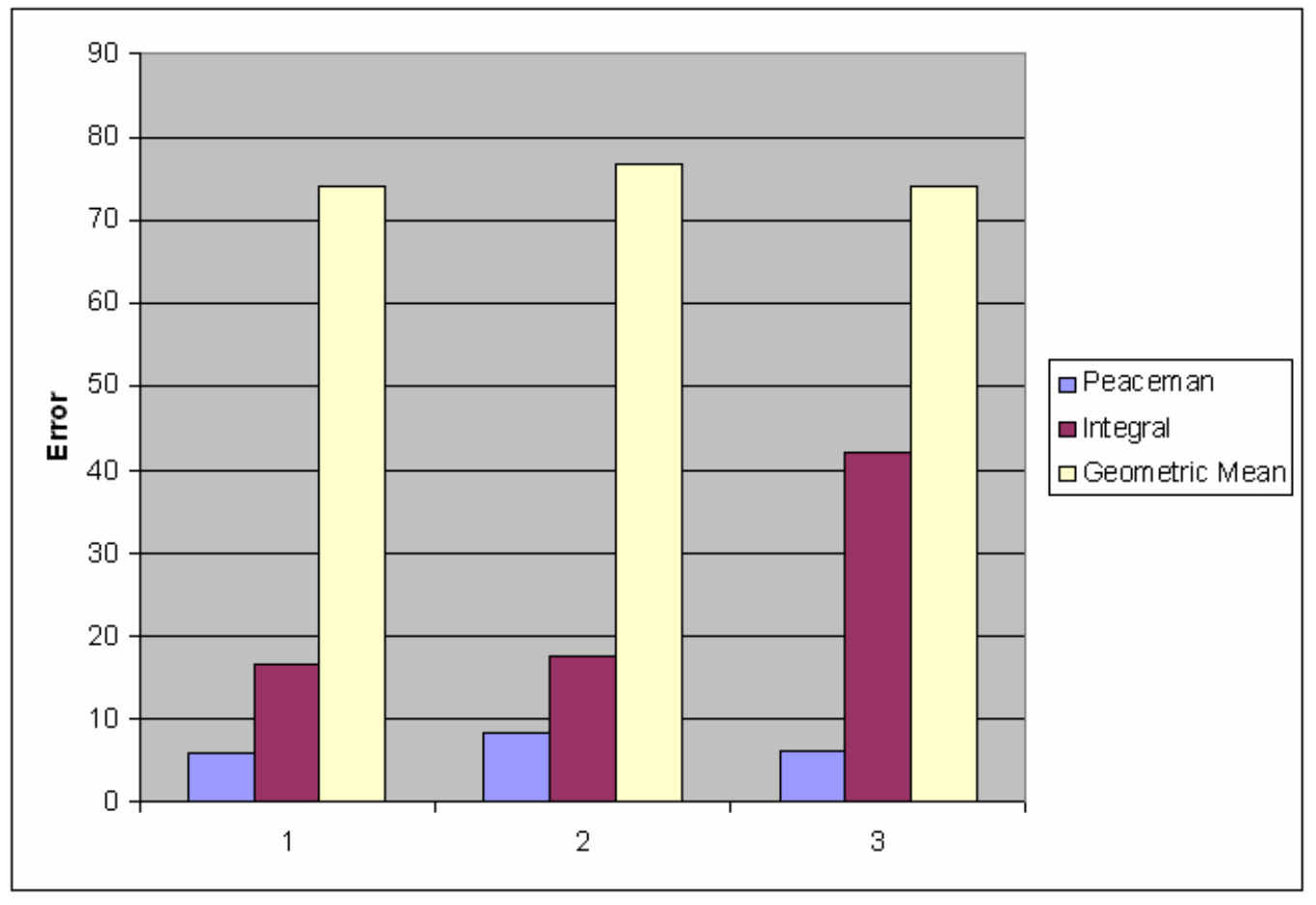

Figure A-9. Results for the various $\ln (r)$ methods 
Table A-1. Constants used in equations (14)-(20)

$$
\begin{aligned}
& A 2 D 2=\frac{1}{k_{A 2}} \ln (3 \Delta x)-\frac{1}{k_{D 2}} \ln \left(r_{w}\right) \\
& A 1 D 2=\frac{1}{k_{A 1}} \ln (3 \Delta x)-\frac{1}{k_{D 2}} \ln \left(r_{\mathrm{w}}\right) \\
& A 0 D 2=\frac{1}{k_{A 0}} \ln (3 \Delta x)-\frac{1}{k_{D 2}} \ln \left(r_{\mathrm{w}}\right) \\
& B 0 D 2=\frac{1}{k_{A 1}} \ln (3 \Delta x)-\frac{1}{k_{D 2}} \ln \left(r_{\mathrm{w}}\right) \\
& D 2 C 2=\left(\frac{1}{k_{D 2}}-\frac{1}{k_{C 2}}\right) \ln \left(\frac{\Delta x^{2}}{4}-\frac{y^{2}}{9}\right)^{\frac{1}{2}} \\
& C 2 B 2=\left(\frac{1}{k_{C 2}}-\frac{1}{k_{B 2}}\right) \ln \left(\frac{9 \Delta x^{2}}{4}+y^{2}\right)^{\frac{1}{2}} \\
& C 2 C 1=\left(\frac{1}{k_{C 2}}-\frac{1}{k_{C 1}}\right) \ln \left(\frac{\Delta x}{2 y}\left(\frac{9 \Delta x^{2}}{4}+y^{2}\right)^{\frac{1}{2}}\right) \\
& B 2 A 2=\left(\frac{1}{k_{B 2}}-\frac{1}{k_{A 2}}\right) \ln \left(\frac{25 \Delta x^{2}}{4}+\frac{25 y^{2}}{9}\right)^{\frac{1}{2}} \\
& B 2 B 1=\left(\frac{1}{k_{B 2}}-\frac{1}{k_{b 1}}\right) \ln \left(\frac{\Delta x}{2 y}\left(\frac{\Delta x^{2}}{4}+y^{2}\right)^{\frac{1}{2}}\right) \\
& A 1 A 1=\left(\frac{1}{k_{B 1}}-\frac{1}{k_{A 1}}\right) \ln \left(\frac{25 \Delta x^{2}}{4}+\frac{25 y^{2}}{9}\right)^{\frac{1}{2}} \\
& A 2 A 1=\left(\frac{1}{k_{A 2}}-\frac{1}{k_{B 1}}\right) \ln \left(\frac{1}{k_{C 1}}-\frac{1}{k_{B 1}}\right) \ln \left(\frac{9 \Delta x^{2}}{4}+y^{2}\right)^{\frac{1}{2}} \\
& A 0 A 1
\end{aligned}
$$




\begin{abstract}
APPENDIX B
This appendix contains a listing of all the files used in this thesis. The files are contained on the CD that is included with this thesis.
\end{abstract}

\title{
Weber
}

This folder contains Hardy's files that were modified to include heterogeneity. The folder Upscale contains the files that solved the pressure coarse grid. The other folders (18Fixed, 54Fixed, 130Fixed, 250Fixed) contain programs that solve for the pressure on a fine grid using the number of nested grid points specified by the folder name. All of the files are written in Fortran 90. The MathCAD file, Perm.xmcd, used to generate the random permeability data sets is also included.

\section{PERM6750v1.txt, PERM6750v2.txt, PERM6750v3.txt}

These files are the three random permeability data sets that were used to test all of the methods in this thesis. They contain 6750 permeabilities which correspond to a simulation reservoir of $15 \times 15 \times 30$ gridblocks.

\section{UpscaleGeometric.xls}

This file contains the upscaling solution using the geometric mean. The different permeability data sets can be read in by editing the ReadK macro. The Run macro loops 
over calculating the sheets effectively iterating the solution. A brief description of the information contained in each tab is presented below.

Krandom - contains the value of the permeability for each cell.

GesselC- contains the upscaling coefficient from the $\ln (\mathrm{r})$ solution.

$\mathrm{Kx}, \mathrm{Ky}, \mathrm{Kz}$ - contain the linking permeabilities in the various directions for the fine grid solution.

Pfine - contains the $15 \times 15 \times 30$ fine grid solution.

Kux, Kuy, Kuz - contain the upscaled linking permeabilities in the various directions for the coarse grid solution.

Pcoarse - contains the $5 \times 5 \times 10$ coarse grid solution.

Errors - tabulates the difference between the two solutions.

\section{Upscalelntegral.xls}

This file contains the upscaling solution using the Integral Method. The same information regarding macros and tabs from UpscaleGeometric.xls file described above, is applicable to this file.

\section{UpscalePeaceman.xls}

This file contains the upscaling solution using the Peaceman Correction. The same information regarding macros and tabs from UpscaleGeometric is applicable to this file. 


\section{Morel-Seytoux.xls}

This file contains the Morel-Seytoux analytical solution to the $5 \times 5 \times 10$ homogeneous reservoir for a given well spacing. Cell N1 is where the well spacing is specified.

\section{Composite.xls}

This file contains the Composite Method solution. Cells F8 and G8 are where the x-position of the wells are specified. The analytical solution for the given well spacing from the file Morel-Seytoux must be pasted into cells C38:L42. The Run macro loops over calculating the sheet which iterates to the solution.

\section{OffcenterwellPeaceman.xls}

This file contains the offcenter well solution that incorporates the Peaceman correction into the well cell. Cells F8 and G8 are where the x-position of the wells are specified. The analytical solution for the given well spacing from the file Morel-Seytoux must be pasted into cells B12:K16. The Run macro loops over calculating the sheet which iterates to the solution. 